\title{
A study of the performance of a generalized exceedance algorithm for detecting noise events caused by road traffic
}

\author{
A. L. Brown ${ }^{\mathrm{a}}$, Bert De Coensel ${ }^{\mathrm{b}, *}$ \\ ${ }^{a}$ School of Environment and Science/Cities Research Institute, Griffith University, Brisbane, Australia \\ ${ }^{b}$ Waves Research Group, Department of Information Technology, Ghent University, Belgium
}

\begin{abstract}
A key question for road traffic noise management is whether prediction of human response to road traffic noise could be improved by accounting for noise events instead of, or in addition to, energy equivalent or percentile measures of noise exposure. However, there is a critical prior question: how should noise events in road traffic be measured? Even at moderate traffic flow rates, detecting and counting noise events caused by road traffic is not a trivial exercise, and as yet there is no generally accepted noise event detection algorithm. This paper investigates the performance of a generalized exceedance algorithm for detecting noise events, constructed on the basis of the literature on noise events caused by road traffic. For this purpose, a microscopic traffic simulation model, coupled to an emission model that accounts for distributions of sound power levels of individual vehicles, is used to simulate one-hour time histories of the noise level in the proximity of a roadway, for an exhaustive set of traffic flow/composition and propagation distance conditions in unshielded locations. The validity and reliability of the number of noise events detected by the generalized algorithm in these one-hour time histories is then evaluated for a range of algorithm parameter sets. By discarding parameter sets that do not result in an algorithm that returns valid or reliable counts, and by examining redundancy in the remaining ones, a small number of representative parameter sets is identified, which may prove useful in the construction of event-based indicators supplementary to energy-equivalent measures of road traffic noise.
\end{abstract}

Keywords: Noise event, Detection algorithm, Indicator, Road traffic

PACS: 43.50.Lj, 43.50.Rq

\section{Introduction}

A noise event in the sound from a stream of road traffic is a discrete component of the sound signal that stands out, or emerges, from the rest of the signal generated by the traffic stream. It is most often the result of the passage of an individual loud vehicle, or succession of vehicles, or even the passage of a not particularly loud vehicle heard against a quieter background in situations of low traffic flow. The term noise event has been used extensively across road, rail and air transport modes (e.g. [1-5]). Synonyms for noise events, and other related terms describing event concepts, are: maxima [6], emergences [7, 8], noise peaks [8], peak or max dB(A) levels [9], or peak noise/sound levels [10-14]. Also used is the non-acoustic term vehicle passby [15]. In the literature of noise events, reference to peaks in road traffic noise signals (as in $[13,16]$ ) are to noise events as described above, not to the peak sound pressure. A related concept, but defined instead as an instant of attention focus by an individual on the physical occurrence of a noise event, is the concept of notice events $[17,18]$. Noise events in traffic noise streams have also been described in inverse, using terms such as lulls [19], noise-free intervals or windows [20].

Noise events in transport noise signals are of interest because of the role they may play in human response to the noise, including disturbance to sleep, annoyance, interference with activities, cardiac responses, and effect on children in schools. Brown [21] has provided an overview of the scattered, but persistent, evidence regarding the effects of noise

${ }^{*}$ Corresponding author

Email addresses: lex.brown@griffith.edu.au (A. L. Brown), bert.decoensel@ugent.be (Bert De Coensel) 
events in road traffic streams on human response. The presence of events is postulated to result in effects beyond those attributed to the level of road traffic noise exposure itself - the latter measured through conventional indicators such as $L_{\mathrm{Aeq}}$ and $L_{\mathrm{A} 10}$. The Environmental Noise Directive [22] notes the potential use of noise events as supplementary to the standardized energy-based indicators of noise exposure, though there appears to be limited application of such supplementary indicators to date. The WHO [23] suggests that events be measured by a combination of number of events and their level, but also noted that as yet there was no generally accepted way to count the number of events. For aircraft noise, measurement of noise events from overflights is standardized (e.g. [24]) and counts of events, or measures such as SEL, are relatively straightforward because of the time separation between successive overflights. However, road traffic can have much shorter vehicle headways, resulting in problematic event detection and counting, even at moderate traffic flow rates [25-27]. Apart from their role in potentially explaining human response to certain traffic noise conditions, there is also an interest in road traffic noise events as an intermediate step in the estimation of energy measures of the whole traffic flow noise signal, or in the estimation of uncertainty measures due to the variability of the acoustic sources in time (as in [28]). The latter applications are not considered further in this paper.

This study applies a rigorous approach to the exploratory definition and measurement of noise events from road traffic-something essential given the largely ad-hoc approaches to event measurement in road traffic noise streams to date. In Section 2 we compile and categorize different formulations of event-based indicators relevant for road traffic noise that have appeared in the literature; formalize a general conceptualization of road traffic noise events based on these; systematically extend the set of event indicators to cover all relevant and realistic exceedance dimensions; and develop a formal approach to the detection of these events in time histories of road traffic noise. We then examine, through a modelling study, the behaviour of this generalized event detection algorithm across acoustic conditions generated from a wide range of traffic parameter and propagation distance conditions found near roadways. The conditions we utilize are, effectively, the population of acoustic conditions that can exist near roadways where there is direct propagation of sound from traffic sources to receivers (that is, excluding only situations with roadside barriers or shielding by urban infrastructure). We also examine the limits on the likely repeatability of measurement of different noise event measures in practice, and the sensitivity of counts of noise events to the parameters of the event detection algorithms that define them. Finally, the wide range of parameter value combinations of the generalized noise event detection algorithm, based on exceedance, is narrowed to a smaller, hence more manageable in future work, valid, reliable set.

The research methodology of the modelling study is outlined in Section 3, and the results are analyzed in Section 4 and Section 5. The intent of this paper is to rigorously and comprehensively report counts of noise events arising from different possible algorithm definitions for detecting noise events in road traffic, the interrelationships between these alternatives, and any practical limitations on their use. Our focus is on alternative ways in which noise events in road traffic noise might be measured, and on the sensitivity of the number of noise events to different traffic and distance conditions for the different formulations of a noise event. Increasing the rigour and the understanding of the occurrence and measurement of road traffic noise events in this way is a prerequisite to future studies that may seek to develop relationships between the level and number of noise events in road traffic streams, and human responses to events in terms of annoyance and sleep disturbance — and to attempts to limit or manage exposure to events.

\section{Noise events}

Estimation of noise event metrics in the sound level signal caused by traffic noise requires a two-step procedure. Firstly, individual events in the time history of the traffic noise signal have to be identified using a detection algorithm based on a set of criteria, for example the instantaneous sound level exceeding a predefined threshold. Secondly, once individual events are identified, summary indicators can be calculated, such as the total number or total duration of all noise events over the period of interest. Sections 2.1 and 2.2 focus on the first stage of identifying noise events based on detection algorithms used in literature over the past decades. These are reviewed, and a generalized noise event detection algorithm is proposed. With events detected by a specific algorithm, noise event metrics for the period of interest can then be calculated. Section 2.3 summarizes different noise event metrics that have been proposed in the same body of literature - though in this paper, the focus is primarily on the number of noise events. 


\subsection{Noise event detection in literature}

A wide range of algorithms, protocols, or criteria, have been reported in the literature for identifying noise events within a time series of A-weighted sound levels-usually from road, rail or aircraft sources. These algorithms have been built into noise measurement equipment systems (e.g. [29]), used in experimentation or field studies (e.g. [30]), or sometimes just postulated as possible approaches to event detection. To date, there is no agreement regarding the appropriate algorithm for event detection in road traffic noise streams. This study makes no a priori assumptions regarding identification of a noise event. Although noise event detection algorithms have typically been constructed $a d$ hoc, a general commonality among algorithms is that they utilize exceedance of some threshold of the instantaneous sound level of the traffic noise signal. Three main categories of algorithms can be identified, which differ in how this threshold is constructed.

In the first category of algorithms, the threshold for noise event detection is set to a predefined, fixed, value. Typical values for this threshold range from $45 \mathrm{~dB}(\mathrm{~A})$ for identifying events in indoor situations with closed windows [31] to $80 \mathrm{~dB}(\mathrm{~A})$ for detecting events in outdoor situations [8, 32]. Some authors [1, 30, 33] have adopted variable thresholds, even within the same study, depending on vehicle flow rates (higher thresholds being used with higher flow rates). In the second category of algorithms, events are detected when the instantaneous sound level emerges by a specified amount above a predefined background level [26, 34, 35]. The first category of algorithms can be seen as a subset of the second category, in which the emergence is set to zero. In the third category, events are detected when the instantaneous sound level emerges by a specified amount, typically 3 to $15 \mathrm{~dB}(\mathrm{~A})$, above another conventional traffic noise indicator, such as $L_{\mathrm{Aeq}}[36,37], L_{\mathrm{A} 50}[7,38]$ or $L_{\mathrm{A} 90}[25,39]$, this way used as an adaptive threshold.

In their suggested event algorithms, most authors have implemented additional decision rules as to whether to retain or reject exceedances above the threshold as noise events. A first criterion commonly encountered is based on the duration of the exceedance. For example, events may have to last for at least 2 to $3 \mathrm{~s}$, before they are counted [1, $25,33,38]$. In [31], a much longer minimum event duration of $30 \mathrm{~s}$ is used, but their detection algorithm is mainly directed towards air and railway traffic noise events. In [29] and [25], in addition, a maximum duration is set on the length of an event. Another criterion is based on the time between events. A minimum time gap between noise events can be set to implement an elementary hysteresis effect into the detection algorithm [25, 29, 39]. This overcomes the problem of multiple event registrations of a single event with irregular rise or decay pattern, and it responds to the (untested) notion that multiple events in short sequence are likely to be perceived as a single event, or that the disturbances caused by multiple events might be experienced as one disturbance.

Table 1 summarizes the parameters for a number of transportation noise event detection algorithms found in literature. This table is not meant to be comprehensive, but gives an idea of the ranges of parameters that have been used in literature, and this has guided the research methodology that will be presented in Section 3 below. For each reference, Table 1 indicates the type of noise source that the algorithm has been designed for, and the situation in which it has been applied (noise events detected indoor or outdoor). Typically, the instantaneous A-weighted sound level envelope of the noise signal is used as the time series, but various time weighting $(\mathrm{S} / \mathrm{F})$ and sampling rates can be found (see [27]). Often not all details were reported as to the basis on which noise events were detected. Note that here, only detection algorithms that are based solely on (the time history of) the sound signal are discussed. There are other approaches, such as those in which event time periods are set a priori based on data from an external database, e.g. for airplane overflights, those in which there is human intervention in selecting events, or those that apply more general pattern recognition techniques to identify outliers (e.g. [40]). These approaches are outside the scope of this work.

\subsection{A generalized noise event detection algorithm}

Based on the overview of the literature in Section 2.1, a generalized noise event detection algorithm based on exceedance is proposed. The approach is illustrated in Figure 1, in which an example of a sound level time history $L_{\mathrm{A}}(t)$ is shown. The onset of the $i$ th noise event at time $t_{1, i}$ is detected when the instantaneous level exceeds a threshold $L_{\alpha}$. This threshold itself consists of a background level $L_{\beta}$ and an emergence $E$,

$$
L_{\alpha}=L_{\beta}+E
$$

and may vary in time (e.g. when the background level $L_{\beta}$ is set to an adaptive value such as $L_{\mathrm{A} 50}$, calculated over a limited period of time). The end $t_{2, i}$ of the noise event is reached when the sound level drops below the threshold $L_{\omega}$. 
Table 1: Various noise event detection algorithms for transportation noise found in literature $\left(L_{\beta}=\right.$ threshold level; $E=$ emergence; $\tau_{e}=$ event duration; $\tau_{g}=$ time between events).

\begin{tabular}{|c|c|c|c|c|c|c|c|}
\hline Reference & Source type & Application & Envelope & $L_{\beta}$ & $E$ & $\tau_{e}$ & $\tau_{g}$ \\
\hline Ribeiro et al. [41] & $\mathrm{road} / \mathrm{rail} / \mathrm{air}$ & outdoor & $L_{\text {Aeq,1s }}$ & $55 \mathrm{~dB}(\mathrm{~A})$ & $0 \mathrm{~dB}(\mathrm{~A})$ & - & - \\
\hline Murray [29] & road & outdoor & $L_{\mathrm{AF}} @ 250 \mathrm{~ms}$ & $65 \mathrm{~dB}(\mathrm{~A})$ & $0 \mathrm{~dB}(\mathrm{~A})$ & $\leq 25 \mathrm{~s}$ & $\geq 3 \mathrm{~s}$ \\
\hline Fidell et al. [33] & air & indoor & $L_{\mathrm{AF}} @ 500 \mathrm{~ms}$ & site-specific & $0 \mathrm{~dB}(\mathrm{~A})$ & $\geq 2 \mathrm{~s}$ & - \\
\hline Sato et al. [42] & road & outdoor & not specified & $75 \mathrm{~dB}(\mathrm{~A})$ & $0 \mathrm{~dB}(\mathrm{~A})$ & - & - \\
\hline Lambert et al. [32] & rail & outdoor & not specified & $70 / 80 \mathrm{~dB}(\mathrm{~A})$ & $0 \mathrm{~dB}(\mathrm{~A})$ & - & - \\
\hline Hall et al. [31] & $\mathrm{road} / \mathrm{rail} / \mathrm{air}$ & indoor & $L_{\mathrm{AS}} @ 1 \mathrm{~s}$ & $45 / 50 / 55 / 60 / 65 \mathrm{~dB}(\mathrm{~A})$ & $0 \mathrm{~dB}(\mathrm{~A})$ & $\geq 30 \mathrm{~s}$ & - \\
\hline Taylor et al. [30] & road/air & outdoor & not specified & $55 / 60 / 65 / 70 / 75 \mathrm{~dB}(\mathrm{~A})$ & $0 \mathrm{~dB}(\mathrm{~A})$ & - & - \\
\hline Fidell et al. [1] & air & indoor/outdoor & $L_{\mathrm{AF}} @ 1 \mathrm{~s}$ & $50 / 60 / 70 \mathrm{~dB}(\mathrm{~A})$ & $0 \mathrm{~dB}(\mathrm{~A})$ & $\geq 2 \mathrm{~s}$ & - \\
\hline Müller et al. [34] & air & indoor/outdoor & $L_{\mathrm{AS}} @ 125 \mathrm{~ms}$ & not specified & $4 \mathrm{~dB}(\mathrm{~A})$ & - & - \\
\hline Mietlicki et al. [35] & road & outdoor & not specified & not specified & $10 \mathrm{~dB}(\mathrm{~A})$ & - & - \\
\hline Griefahn et al. [26] & $\mathrm{road} / \mathrm{rail}$ & indoor & not specified & not specified & $10 \mathrm{~dB}(\mathrm{~A})$ & - & - \\
\hline Wunderli et al. [36] & $\mathrm{road} / \mathrm{rail} / \mathrm{air}$ & outdoor & $L_{\text {Aeq,1s }}$ & $L_{\text {Aeq, }}$ & $3 \mathrm{~dB}(\mathrm{~A})$ & - & - \\
\hline Campbell and Isles [37] & road & outdoor & $L_{\mathrm{Aeq}, 250 \mathrm{~ms}}$ & $L_{\text {Aeq }}$ & $15 \mathrm{~dB}(\mathrm{~A})$ & - & - \\
\hline De Coensel and Botteldooren [38] & $\mathrm{road} / \mathrm{rail} / \mathrm{air}$ & outdoor & $L_{\mathrm{AS}} @ 1 \mathrm{~s}$ & $L_{\mathrm{A} 50,30 \mathrm{~s}}$ & $3 \mathrm{~dB}(\mathrm{~A})$ & $\geq 3 \mathrm{~s}$ & - \\
\hline Aasvang et al. [25] & $\mathrm{road} / \mathrm{rail}$ & indoor/outdoor & $L_{\mathrm{AF}} @ 1 \mathrm{~s}$ & $L_{\mathrm{A} 90,5 \min }$ & $10 \mathrm{~dB}(\mathrm{~A})$ & $\geq 2 \mathrm{~s}, \leq 40 \mathrm{~s}$ & $\geq 5 \mathrm{~s}$ \\
\hline Tulen et al. [39] & road & indoor & $L_{\text {Aeq, } 1 \mathrm{~s}}$ & $L_{\mathrm{A} 90,10 \mathrm{~min}}$ & $10 \mathrm{~dB}(\mathrm{~A})$ & - & $\geq 15 \mathrm{~s}$ \\
\hline
\end{tabular}

Other characteristics of the $i$ th noise event are its duration $\tau_{e, i}=t_{2, i}-t_{1, i}$, the time gap (or noise free interval) since the previous noise event $\tau_{g, i}=t_{1, i}-t_{2, i-1}$, the maximum sound level $L_{\mathrm{Amax}, i}$, the time $t_{\mathrm{max}, i}$ when this maximum sound level within the event is reached, and its event sound exposure level

$$
\mathrm{SEL}_{e, i}=10 \log _{10}\left(\int_{t_{1, i}}^{t_{2, i}} 10^{L_{\mathrm{A}}(t) / 10} \mathrm{~d} t\right)
$$

These additional characteristics can be used to retain/reject particular events. Table 1 shows the values for the parameters $L_{\beta}$ and $E$, and the ranges for the parameters $\tau_{e}$ and $\tau_{g}$ used in the references.

\subsection{Summary metrics based on detected noise events}

Once individual noise events are detected, summary indicators can be calculated over a given period of interest. Table 2 gives an overview of some summary metrics that others have proposed (again, this list is not meant to be exhaustive). According to Marks et al. [43], summary metrics can be categorized into those that characterize the microstructure or the macrostructure of the sound signal time history. The former characterize the properties of individual events whereas the latter characterize the succession of noise events. These categories are separated in Table 2 by a horizontal line. In practice, for road traffic noise, both categories depend on quite different physical drivers. The acoustical microstructure of noise events depends mainly on vehicle type and source strength of the vehicles on the roadway, on the speed at which they are travelling, and on the distance from the roadway sources to the receiver. By contrast, the acoustical macrostructure depends mainly on the volume of traffic on the roadway.

By far the most commonly used macroscopic metric is simply the number $N$ (also noted as $N N E$ ) of noise events over a period of interest with duration $T$. This number can be normalized to a particular time unit, such as the number of events per hour, and can be counted in bins according to the maximum level of the noise event. Referring to the notion of the threshold that is used to detect noise events, this metric is also sometimes called the "number above threshold" NA. A second metric is the total (cumulative) duration $T_{e}$ of noise events, sometimes called "time above threshold" $T A$. A related metric is the mask index $M I$, defined as the fraction of time that noise events are detected [8]. In contrast, one may also consider the noise free intervals as the relevant metric (see e.g. Roberts et al. [19]), and define the total and average duration of noise free intervals (or the "time below threshold" $T B$ ).

Examples of microscopic metrics are the average and worst case maximum level of noise events, the average rise time of noise events, or the total sound exposure level of noise events, defined in Table 2. Two additional metrics are the intermittency ratio $I R$ [36] and the unexpectancy $U$ [44]. The intermittency ratio is defined as the ratio of the 


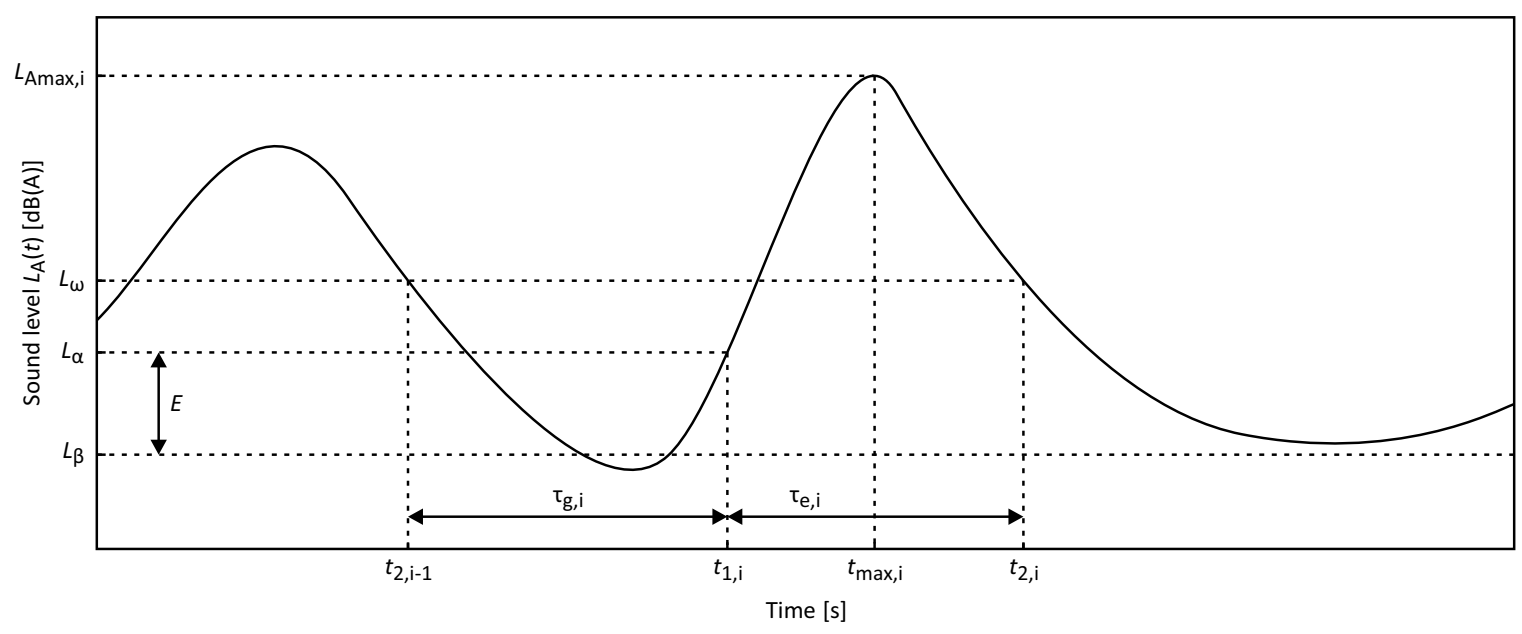

Figure 1: Parameters of the generalized sound event detection algorithm.

event-based sound energy to the total sound energy in the traffic noise signal. The possibility of calculating energyequivalent sound level metrics on only that part of the sound signal above some threshold has been suggested several times in the past $[18,45]$. The unexpectancy is defined as a linear combination of the variance of the maximum levels of the sequence of events and the variance of the temporal gap between successive events, with $a$ and $b$ being constants (no values are given in [44]). The rationale behind this metric is the hypothesis that when events are regular in succession, such as with trains passing by, they are much more predictable, whereas for irregular events such as originating from air or road traffic, they are not expected.

In this paper, only the macrostructure summary metric, number of noise events, will be considered.

\section{Methodology}

The overall methodology involves testing how different formulations of event detection perform across the population of acoustic conditions that exist near roadways. A modelling approach was adopted as this provides the ability to test the generalized algorithm against time histories of traffic noise generated for all likely situations. In these, traffic flow variables, and the propagation distance from roadway to receiver, covered a full range of realistic values, and these could be varied independently - something that would be largely impractical using data gathered in field measurement studies. The modelling study follows a two-stage approach. Firstly, the time history of instantaneous sound level is simulated for a large set of road traffic noise exposure scenarios (Sections 3.1, 3.2 and 3.3). Secondly, an extensive set of alternative parameter values of the generalized exceedance algorithm (Section 3.4) is utilized to detect and count noise events in these time histories. Analysis focuses on the repeatability of the counts of events when using different parameter values in the exceedance algorithm, and on examining, and reducing, redundancy in the results obtained from the many alternative formulations of event detection.

\subsection{Simulation of instantaneous sound level}

The instantaneous sound level in free field caused by road traffic is simulated using the road traffic noise pattern simulation model described in De Coensel et al. [46]. This model, called Noysim2, combines a microscopic simulation of road traffic with an instantaneous vehicle noise emission and a point-to-point sound propagation model. Aimsun, a commercially available microscopic road traffic simulation model (distributed by TSS Transport Simulation Systems), is used to simulate the traffic. In particular, given a road network, vehicle fleet properties and aggregated traffic demand data, the movement of individual vehicles is simulated, and the instantaneous position, speed and acceleration of each vehicle at each timestep during a predefined simulation period is provided.

Subsequently, the instantaneous emission of all sources is calculated using a noise emission model that includes distributions of vehicle sound power levels [46]. This model is based on the Imagine road traffic noise emission 
Table 2: Overview of macrostructure and microstructure summary metrics of noise events.

\begin{tabular}{|c|c|c|}
\hline Description & Formula & \\
\hline Total number of noise events & $N$ & \\
\hline Total duration of noise events & $T_{e}=\sum_{i=1}^{N} \tau_{e, i}$ & \\
\hline Average duration of noise events & $\overline{T_{e}}=\frac{T_{e}}{N}$ & \\
\hline Mask index [8] & $M I=\frac{T_{e}}{T}$ & \\
\hline Total duration of noise free intervals & $T_{g}=\sum_{i=1}^{N} \tau_{g, i}$ & \\
\hline Average duration of noise free intervals & $\overline{T_{g}}=\frac{T_{g}}{N}$ & \\
\hline Average maximum level of noise events & $\overline{L_{\mathrm{Amax}}}=\frac{1}{N} \sum_{i=1}^{N} L_{\mathrm{Amax}, \mathrm{i}}$ & \\
\hline Worst case maximum level of noise events & $\max _{i}\left\{L_{\mathrm{Amax}, \mathrm{i}}\right\}$ & \\
\hline Average rise time of noise events & $\frac{1}{N} \sum_{i=1}^{N} \frac{L_{\mathrm{Amax}, \mathrm{i}}-L_{\alpha}}{t_{\max , \mathrm{i}}-t_{1, \mathrm{i}}}$ & \\
\hline Total sound exposure level of noise events & $\mathrm{SEL}_{e}=10 \log _{10} \sum_{i=1}^{N} 10^{\mathrm{SEL}_{e, i} / 10}$ & \\
\hline Intermittency ratio [36] & $I R=\frac{100}{T} \frac{10^{\mathrm{SEL}_{e} / 10}}{10^{L_{\mathrm{Aeq}, \mathrm{T}} / 10}}$ & \\
\hline Unexpectancy [44] & $U=a \cdot \operatorname{var}_{i}\left\{L_{\mathrm{Amax}, \mathrm{i}}\right\}+b \cdot \log _{10}$ & $\left(\frac{\underset{j}{\operatorname{var}\left\{\tau_{g, j}\right\}}}{T}\right)$ \\
\hline
\end{tabular}

model [47], to which a per-vehicle correction is added that accounts for the distribution in sound power emitted by individual vehicles within different categories. Correction distributions were constructed based on a large set of roadside measurements [48]. Using individualized vehicle emission laws, this road traffic noise emission model accounts for the influence of vehicles that are producing more/less noise than the average vehicle.

Finally, the output of the Noysim 2 road traffic noise simulation model consists of the time history of the instantaneous sound level at the location of the receiver, calculated in 1/3-octave bands using the ISO 9613 sound propagation model [49]. As only broadband road traffic noise exposure is considered in this work, all sources have a similar emission spectrum. Spectral aspects are therefore neglected in first order, and only the A-weighted instantaneous sound level at the receiver is considered. Psychoacoustic effects such as frequency masking, and the differential effect on the frequency spectrum of transmission of the road traffic noise signals through building envelope components, are thus outside the scope of this work. More details about the Noysim 2 model and its operation can be found in De Coensel et al. [46].

\subsection{Noise exposure scenarios}

The modelling is based on a receiver adjacent to a straight dual-lane roadway carrying uninterrupted traffic. This is a setting encountered in rural areas, but also in urban areas away from intersections or signal controls. A wide range of road traffic noise exposure scenarios were modelled, even for this simple geometry, by varying traffic flow variables and the distance between the receiver and the road. Figure 2 shows a schematic drawing of the setting, together with the values used in the construction of the scenarios. 


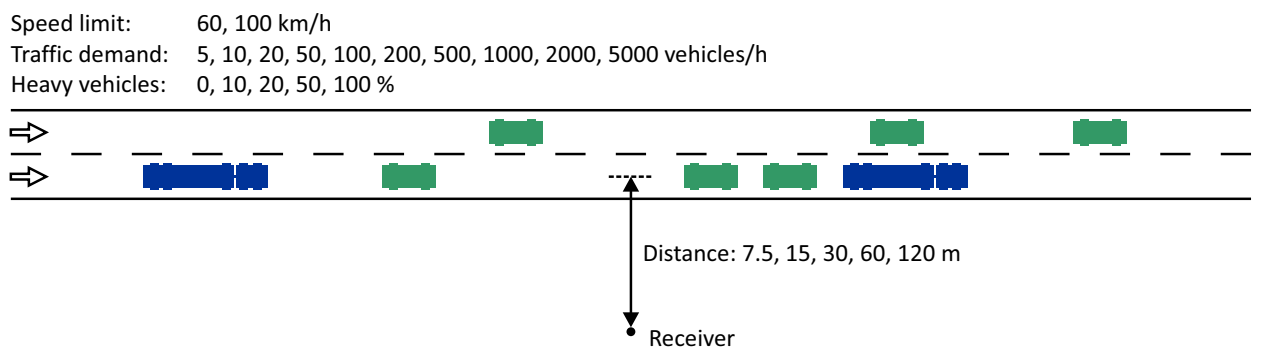

Figure 2: Schematic drawing of the simulated setting, together with variable value ranges.

By using a broad range for each of the variables, the idea is that the modelled scenarios include the population of noise conditions that could occur, in reality, along a single road link. An exhaustive set of scenarios is created by varying the speed limit $(60 \mathrm{~km} / \mathrm{h}$ or $100 \mathrm{~km} / \mathrm{h}$ ), the traffic demand (from 5 to 5000 vehicles $/ \mathrm{h}$ ), the percentage of heavy vehicles (from $0 \%$ to $100 \%$ ), and the distance to the road (from $7.5 \mathrm{~m}$ to $120 \mathrm{~m}$ ), as shown in Figure 2 . The total number of unique scenarios thus equals $2 \times 10 \times 5 \times 5=500$. This simplified geometry is adequate for the purpose of this study, but more complex road network configurations could be modelled by the same system, where multiple roadway links, roadway intersections, and signalized controls on traffic could be introduced. The analysis is restricted to uninterrupted flow road traffic streams, in contrast to situations where traffic is congested or is known to be controlled by traffic signals or other devices (as in [8]). A rigorous investigation into the influence of different parameter values of the generalized algorithm for the detection of noise events from freely flowing road traffic streams is a prerequisite to any later application to interrupted traffic flow conditions. Note that the modelled settings also consider only unobstructed propagation, excluding situations with roadside barriers or shielding by urban infrastructure. We could have included the latter in our modelling, but as their effect is to reduce the emergence of peaks of passing vehicles above roadway background levels at receivers, obstructed propagation scenarios would not add unique dimensions to the population of noise scenarios that were modelled in this study.

The duration of each simulation was set at $1 \mathrm{~h}$, with a timestep of $125 \mathrm{~ms}$. The selected timestep allows for a good temporal resolution of the simulations, and equals the shortest sample interval found in the literature overview in Section 2.1. The choice of simulation duration was chosen as a compromise between simulation variability and real-life measurement pragmatics. On the one hand, variability between simulation runs can be expected due to the stochastic nature of the simulation model, and this variability is reduced by increasing the simulation duration. On the other hand, eventual field measurements based on the generalized event detection algorithm being examined, or potential future application of number of noise events as a noise limit, require that the measurement duration is kept within practical limits, and a $1 \mathrm{~h}$ period for these seems appropriate. The actual $1 \mathrm{~h}$ simulation runs included an additional warm-up period of 5 minutes. Vehicles are loaded onto the network at the entry of the road, randomly distributed in time according to a negative exponential distribution.

The simulation for each unique scenario was repeated 30 times, resulting in a total of 15,000 simulation runs (30 $\times 500$ ) of $1 \mathrm{~h}$ time histories of road traffic noise levels near the roadway.

\subsection{Building attenuation and background level}

In general, standards and criteria for road, rail and air traffic noise are specified as limit levels outside of buildings (either free field, or as incident on or near the external façade). Most of these limits originated from exposure-effect studies in which exposure was measured outside of dwellings, and people's annoyance, or some other effect, assessed whilst inside the dwelling. All predictions, mapping of exposure, assessment and (most) management of transportation noise is thus performed using outside noise levels, but with the outside noise level limits set to manage indoor human response.

There is a major difference in the consideration of noise events: most laboratory or field studies of sleep disturbance have used noise stimuli specified in terms of levels at the sleeper's ear-that is indoor levels. Thus any future limit criteria for noise events is appropriately based on indoor levels. For this study, in order to relate the simulated outdoor noise level time histories to noise exposure limits for sleep disturbance based on indoor levels, outdoor 
time histories have to be modified by the attenuation provided by building façades (obviously façade attenuation is frequency dependent, but for present purposes a consideration of A-weighted attenuations is sufficient). Outside-toinside attenuation of dwellings is critically dependent on the state of closure of windows in the sleeping room and, if closed, window size and sound reduction of the window. Furthermore, human behavior with respect to window operations significantly alters the level of attenuation, and there is likely a different behavior between summer and winter, tropical and temperate climates, air-conditioned or heated premises etc. There are divergent estimates of the effect of the building envelope and of the state of window closure on the outdoor/indoor difference in levels of noise event maxima. Marks et al. [43], assuming tilted windows, applied a $15 \mathrm{~dB}(\mathrm{~A})$ attenuation to outdoor free-field measured noise levels they presented to sleepers indoors. Measurements by Aasvang et al. [25] showed mean attenuation values of $26 \mathrm{~dB}(\mathrm{~A})$ for closed windows, and 11 to $24 \mathrm{~dB}(\mathrm{~A})$ for open windows. Müller et al. [34] found median attenuation values of 14,18 and $28 \mathrm{~dB}(\mathrm{~A})$ for open, tilted and closed windows respectively. The Night Noise Guidelines for Europe [23] adopts an average insulation value of $21 \mathrm{~dB}(\mathrm{~A})$ for outside to inside attenuation, noting that a large part of the population in Europe sleeps with windows slightly open.

In addition to estimating the time history of road traffic noise indoors by accounting for the building envelope attenuation, there is a need for additional consideration of the background levels experienced indoors, i.e. the levels against which noise events from passing vehicles emerge. In urban areas, the outdoor background signal is likely to originate from traffic on the road network at most hours of the day, except during the late night/very early morning hours of low urban traffic flow. At those times, the background signal may be from insects or other natural sounds, as would be the case in lower trafficked urban or rural areas. By contrast, the indoor background signal may additionally be set by internally generated sources within the building, such as refrigerators, clocks, ventilator fans or similar. Both building envelope attenuation and background level have an effect on the emergence of noise events, and consequently, the outcome of any algorithm that counts noise events based on their emergence from the background.

In order to address the application of noise event detection algorithms to assess indoor human response to noise, the parameter study performed in this work is based on the indoor sound. For the purposes of this study, this is illustrated by considering just two conditions for building envelope attenuations: fully open and fully closed windows. Using the mixed evidence described above, an outside to inside attenuation of $5 \mathrm{~dB}(\mathrm{~A})$ is applied for the windows open condition. For the closed windows, a building insulation of $25 \mathrm{~dB}(\mathrm{~A})$ is used. Additionally, to simulate the indoor time history, an internally generated level of $35 \mathrm{~dB}(\mathrm{~A})$ is added to the simulated road traffic time history experienced indoors. It can be foreshadowed that this internally generated level of $35 \mathrm{~dB}(\mathrm{~A})$ only affects those scenarios which generate relatively low noise exposures, i.e. those with low traffic volumes or at relatively large distance from the road, and for all closed window conditions.

We thus base this examination of the performance of the generalized noise event detection algorithm for different parameter values on building-attenuated time histories of road traffic noise signals as they would be experienced indoors. We do note, however, that for longer-term practical application within the current paradigm of noise management based on outside levels, the specific limits on indoor noise event levels (e.g. for sleep protection), would require translation to an equivalent outdoor event limit.

\subsection{Noise event detection algorithm parameter ranges}

For each of the simulated scenarios, and for both building envelope conditions considered, noise event counts are generated using the generalized noise event detection algorithm outlined in Section 2.2, by varying two main parameters of the algorithm: the detection threshold $L_{\alpha}$ and the minimum time gap $\tau_{g}$ between events. For the detection threshold, both the fixed and adaptive case is considered. Fixed thresholds vary from 45 to $75 \mathrm{~dB}(\mathrm{~A})$ in steps of $5 \mathrm{~dB}(\mathrm{~A})$ (in a pilot study, also higher limits of 80,85 and $90 \mathrm{~dB}(\mathrm{~A})$ were considered, but such high thresholds did not lead to results that were repeatable and/or sensitive to changes in traffic parameter or distance conditions). The adaptive thresholds considered apply $L_{\mathrm{Aeq}}, L_{\mathrm{A} 50}$ or $L_{\mathrm{A} 90}$ as background level $L_{\beta}$, and $3,5,10$ and $15 \mathrm{~dB}(\mathrm{~A})$ as minimum emergences $E$ above $L_{\beta}$. In this study, the adaptive thresholds $L_{\mathrm{Aeq}}, L_{\mathrm{A} 50}$ and $L_{\mathrm{A} 90}$ are calculated over the full $1 \mathrm{~h}$ simulation duration; in continuous measurement applications, one could consider to use a moving time window. Four alternatives were used for the minimum time gap between events: 3, 5, 10 or 30 s. No lower limit was set on the duration of events since the simulated sound level time histories only contain road traffic noise, and there was no need to filter out occasional spikes of non-traffic flow related events such as door slams. This resulted in 76 different parameter sets (19 combinations of $L_{\beta}$ and $E$, with 4 values of minimum time gap for each). Note that this selection covers to some extent the redundancy in using emergence criteria in association with fixed threshold criteria; 
Table 3: Naming convention for the 76 different parameter sets of the generalized noise event detection algorithm used in the modelling.

\begin{tabular}{|c|c|c|c|c|c|}
\hline \multirow[t]{2}{*}{ Threshold } & \multirow[t]{2}{*}{$L_{\alpha}[\mathrm{dB}(\mathrm{A})]$} & \multicolumn{4}{|c|}{ Minimum time gap between noise events } \\
\hline & & $\tau_{g}=3 \mathrm{~s}$ & $5 \mathrm{~s}$ & $10 \mathrm{~s}$ & $30 \mathrm{~s}$ \\
\hline \multirow[t]{7}{*}{ Fixed } & 45 & T45E00G03 & T45E00G05 & T45E00G10 & T45E00G30 \\
\hline & 50 & T50E00G03 & T50E00G05 & T50E00G10 & T50E00G30 \\
\hline & 55 & T55E00G03 & T55E00G05 & T55E00G10 & T55E00G30 \\
\hline & 60 & T60E00G03 & T60E00G05 & T60E00G10 & T60E00G30 \\
\hline & 65 & T65E00G03 & T65E00G05 & T65E00G10 & T65E00G30 \\
\hline & 70 & T70E00G03 & T70E00G05 & T70E00G10 & T70E00G30 \\
\hline & 75 & T75E00G03 & T75E00G05 & T75E00G10 & T75E00G30 \\
\hline \multirow{12}{*}{ Adaptive } & $L_{\text {Aeq }}+3$ & LEQE03G03 & LEQE03G05 & LEQE03G10 & LEQE03G30 \\
\hline & $L_{\text {Aeq }}+5$ & LEQE05G03 & LEQE05G05 & LEQE05G10 & LEQE05G30 \\
\hline & $L_{\text {Aeq }}+10$ & LEQE10G03 & LEQE10G05 & LEQE10G10 & LEQE10G30 \\
\hline & $L_{\text {Aeq }}+15$ & LEQE15G03 & LEQE15G05 & LEQE15G10 & LEQE15G30 \\
\hline & $L_{\mathrm{A} 50}+3$ & L50E03G03 & L50E03G05 & L50E03G10 & L50E03G30 \\
\hline & $L_{\mathrm{A} 50}+5$ & L50E05G03 & L50E05G05 & L50E05G10 & L50E05G30 \\
\hline & $L_{\mathrm{A} 50}+10$ & L50E10G03 & L50E10G05 & L50E10G10 & L50E10G30 \\
\hline & $L_{\mathrm{A} 50}+15$ & L50E15G03 & L50E15G05 & L50E15G10 & L50E15G30 \\
\hline & $L_{\mathrm{A} 90}+3$ & L90E03G03 & L90E03G05 & L90E03G10 & L90E03G30 \\
\hline & $L_{\mathrm{A} 90}+5$ & L90E05G03 & L90E05G05 & L90E05G10 & L90E05G30 \\
\hline & $L_{\mathrm{A} 90}+10$ & L90E10G03 & L90E10G05 & L90E10G10 & L90E10G30 \\
\hline & $L_{\mathrm{A} 90}+15$ & L90E15G03 & L90E15G05 & L90E15G10 & L90E15G30 \\
\hline
\end{tabular}

e.g. an emergence of $E=5 \mathrm{~dB}(\mathrm{~A})$ above $L_{\beta}=45 \mathrm{~dB}(\mathrm{~A})$ is identical to an emergence of $E=0 \mathrm{~dB}(\mathrm{~A})$ above $L_{\beta}=$ $50 \mathrm{~dB}(\mathrm{~A})$.

Table 3 shows the naming convention adopted for the parameter sets of the generalized event detection algorithm utilized; for convenience, the number of noise events obtained through application of the generalized algorithm with a particular parameter set is identified below by the same label as the parameter set itself. For example, the label LEQE10G03 denotes the outcome (i.e. the number of events) of the algorithm that detects the occurrence of noise events that emerge at least $10 \mathrm{~dB}(\mathrm{~A})$ above $L_{\mathrm{Aeq}}$, with a minimum time gap of $3 \mathrm{~s}$ between successive noise events. To clarify that the algorithm is applied to the sound level simulated indoors with fully open or closed windows, the suffix 'OP' or 'CL' is added to the variable name.

Figure 3 provides illustrations of the application of the complete sound level simulation and event detection methodology, for one of the 30 instances simulated for one of the 500 available scenarios. The generalized event detection algorithm is illustrated with two parameter sets, each applied to both the window open and closed situations (i.e. T60E00G03OP and T60E00G03CL; LEQE05G03OP and LEQE05G03CL). The outdoor sound level time history is shown in a solid green line, whereas the indoor sound level time history is shown in a solid blue line, for both open and closed windows (only the first 10 minutes of the simulation are shown). The figure illustrates the detection of noise events, as vertical red bars, through two different detection algorithm settings, one with a fixed threshold at $60 \mathrm{~dB}(\mathrm{~A})$ (detecting 1 or 0 noise events for open and closed window conditions respectively), the other with an adaptive threshold at $L_{\mathrm{Aeq}}+5 \mathrm{~dB}(\mathrm{~A})$ (detecting 4 or 1 noise events for open and closed window conditions respectively).

\section{Basic performance of the generalized algorithm}

\subsection{Validity}

Selection of appropriate parameter values for the road traffic noise event detection algorithm from the large set of alternatives considered in this work will eventually have to be assessed through human effects research - that is, by examining if and how different noise event measures correlate with human outcomes (sleep disturbance, annoyance etc.). However, there are also a priori criteria that any event detection algorithm must meet, in terms of validity (is the number of events detected reasonable, and does the algorithm differentiate well between different conditions?) 

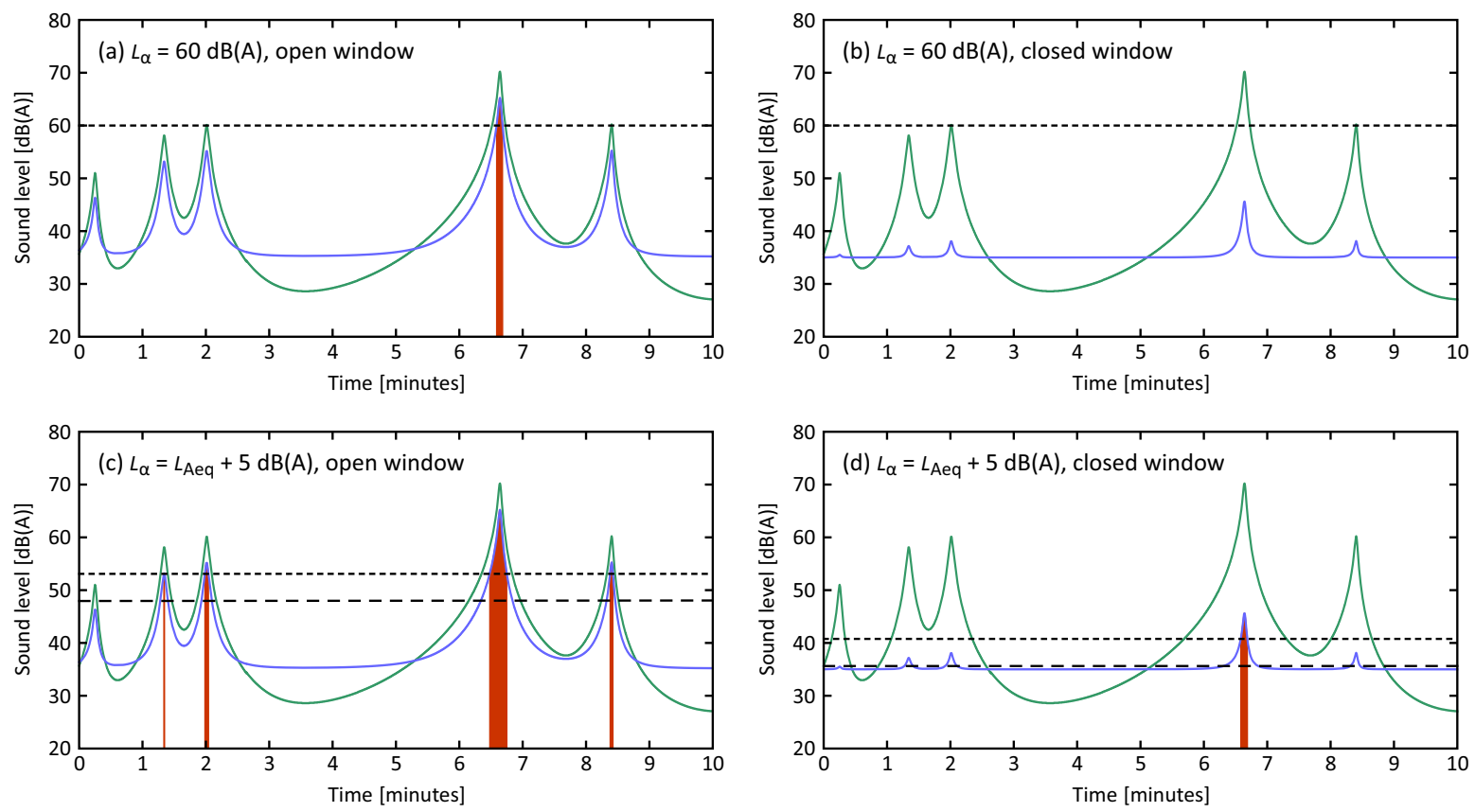

Figure 3: Simulated time history of the outdoor sound level (free-field, solid green line) at a distance of $30 \mathrm{~m}$ from a two-lane road with a speed limit of $60 \mathrm{~km} / \mathrm{h}$, and a traffic volume of 50 vehicles $/ \mathrm{h}$, of which $20 \%$ heavy vehicles. The indoor sound level time history is shown in a solid blue line, for open windows on panels (a) and (c), for closed windows on panels (b) and (d). The detection of noise events within the indoor sound level time history is illustrated using two algorithm settings, on panels (a) and (b) with $L_{\alpha}$ set to $60 \mathrm{~dB}(\mathrm{~A})$ (short dashed line), and on panels (c) and (d) with $L_{\alpha}$ set to $L_{\mathrm{Aeq}}+5 \mathrm{~dB}(\mathrm{~A})$ (long dashed line represents $L_{\mathrm{Aeq}}$ ). In both algorithm settings, the minimum time gap between events is set to $3 \mathrm{~s}$. Detected noise events are tagged using a vertical red bar, for the duration of the event. 
Table 4: Mean of the number of noise events per hour detected by the generalized algorithm, averaged over the 500 scenarios, for each parameter set considered. The numbers in parentheses show the standard deviation of the number of noise events per hour detected in the 30 simulation replications of each scenario, again averaged across the 500 scenarios. The cells shaded in red indicate parameter sets that may not result in the detection of a sufficient number of events and therefore show low validity (see Section 4.1); the cells shaded in blue indicate parameter sets that result in low reliability (see Section 4.2).

\begin{tabular}{|c|c|c|c|c|c|c|c|c|c|}
\hline \multirow[t]{2}{*}{ Threshold } & \multirow[t]{2}{*}{$L_{\alpha}[\mathrm{dB}(\mathrm{A})]$} & \multirow{2}{*}{$\begin{array}{c}\text { Open window } \\
\tau_{g}=3 \mathrm{~s}\end{array}$} & \multicolumn{7}{|c|}{ Closed window } \\
\hline & & & $5 \mathrm{~s}$ & $10 \mathrm{~s}$ & $30 \mathrm{~s}$ & $\tau_{g}=3 \mathrm{~s}$ & $5 \mathrm{~s}$ & $10 \mathrm{~s}$ & $30 \mathrm{~s}$ \\
\hline \multirow[t]{7}{*}{ Fixed } & 45 & $15.7(2.8)$ & $15.3(2.7)$ & $13.9(2.4)$ & $9.3(1.8)$ & $43.1(4.9)$ & $34.0(4.1)$ & $22.6(3.3)$ & $10.3(2.2)$ \\
\hline & 50 & $22.5(3.6)$ & $21.1(3.4)$ & $17.8(2.8)$ & $10.6(1.9)$ & $37.1(4.4)$ & $28.4(3.8)$ & $18.4(2.9)$ & $8.4(2.0)$ \\
\hline & 55 & $31.1(4.4)$ & $27.7(4.0)$ & $21.2(3.3)$ & $11.2(2.1)$ & $24.0(3.5)$ & $18.8(3.1)$ & $12.6(2.5)$ & $6.0(1.8)$ \\
\hline & 60 & $39.9(4.6)$ & $33.1(4.1)$ & $23.3(3.4)$ & $11.1(2.2)$ & $10.3(2.5)$ & $8.7(2.2)$ & $6.4(1.9)$ & $3.5(1.4)$ \\
\hline & 65 & $43.0(4.9)$ & $33.8(4.1)$ & $22.4(3.3)$ & $10.2(2.2)$ & $2.5(1.4)$ & $2.3(1.3)$ & $2.1(1.1)$ & $1.5(0.9)$ \\
\hline & 70 & $36.9(4.4)$ & $28.3(3.8)$ & $18.3(3.0)$ & $8.3(2.0)$ & $0.5(0.7)$ & $0.5(0.6)$ & $0.5(0.6)$ & $0.4(0.6)$ \\
\hline & 75 & $24.0(3.5)$ & $18.8(3.1)$ & $12.6(2.5)$ & $6.0(1.8)$ & $0.1(0.3)$ & $0.1(0.3)$ & $0.1(0.3)$ & $0.1(0.3)$ \\
\hline \multirow[t]{12}{*}{ Adaptive } & $L_{\mathrm{Aeq}}+3$ & $60.6(9.4)$ & $54.2(7.8)$ & $42.8(5.8)$ & $22.5(3.7)$ & $54.0(8.5)$ & $48.0(6.9)$ & $37.4(5.0)$ & $19.1(3.2)$ \\
\hline & $L_{\text {Aeq }}+5$ & $42.1(8.2)$ & $39.2(7.1)$ & $33.5(5.5)$ & $20.6(3.5)$ & $35.3(7.1)$ & $32.7(6.1)$ & $27.7(4.6)$ & $16.5(2.9)$ \\
\hline & $L_{\text {Aeq }}+10$ & $14.8(4.3)$ & $14.5(4.2)$ & $13.8(3.8)$ & $11.5(2.9)$ & $10.4(3.3)$ & $10.2(3.2)$ & 9.7 (2.9) & $8.0(2.2)$ \\
\hline & $L_{\mathrm{Aeq}}+15$ & $4.8(2.1)$ & $4.8(2.1)$ & $4.7(2.0)$ & $4.4(1.8)$ & $2.7(1.4)$ & $2.7(1.4)$ & $2.6(1.4)$ & $2.5(1.3)$ \\
\hline & $L_{\mathrm{A} 50}+3$ & $76.9(6.1)$ & $63.0(5.2)$ & $43.2(4.2)$ & $19.0(2.6)$ & $71.8(5.6)$ & $58.4(4.8)$ & $39.6(3.9)$ & $17.6(2.5)$ \\
\hline & $L_{\mathrm{A} 50}+5$ & $65.5(5.6)$ & $56.1(5.0)$ & $41.1(4.1)$ & 19.7 (2.6) & $56.6(5.0)$ & $48.0(4.5)$ & 34.5 (3.7) & $16.2(2.5)$ \\
\hline & $L_{\mathrm{A} 50}+10$ & $37.1(4.1)$ & $34.4(3.9)$ & $28.9(3.4)$ & $17.2(2.5)$ & $24.9(3.4)$ & $22.9(3.2)$ & $19.1(2.9)$ & $11.3(2.2)$ \\
\hline & $L_{\mathrm{A} 50}+15$ & $20.1(2.8)$ & $19.3(2.7)$ & $17.4(2.6)$ & $12.3(2.1)$ & $9.1(2.1)$ & $8.8(2.0)$ & $8.0(2.0)$ & $6.0(1.7)$ \\
\hline & $L_{\mathrm{A} 90}+3$ & $53.4(7.4)$ & $40.6(5.9)$ & $25.4(4.0)$ & $10.2(2.0)$ & $65.0(6.5)$ & $50.4(5.3)$ & 32.9 (3.9) & $14.2(2.4)$ \\
\hline & $L_{\mathrm{A} 90}+5$ & $65.2(8.1)$ & $50.5(6.6)$ & $32.9(4.6)$ & $14.2(2.4)$ & $66.4(6.3)$ & $51.4(5.2)$ & $33.3(4.0)$ & $14.7(2.4)$ \\
\hline & $L_{\mathrm{A} 90}+10$ & $60.8(6.9)$ & $50.9(6.0)$ & $36.0(4.7)$ & $17.0(2.7)$ & $44.4(4.8)$ & $36.8(4.2)$ & $25.6(3.5)$ & $11.8(2.3)$ \\
\hline & $L_{\mathrm{A} 90}+15$ & $40.8(5.5)$ & $36.8(4.9)$ & $29.4(4.1)$ & $15.9(2.6)$ & $19.5(3.4)$ & $17.7(3.1)$ & $14.4(2.7)$ & $8.1(2.0)$ \\
\hline
\end{tabular}

and reliability (does the algorithm produce consistent counts of number of noise events when applied to identical conditions?).

Table 4 shows the mean number of noise events detected by the generalized algorithm using each of the 152 parameter sets in Table 3 (76 open-window and 76 closed-window) in the time histories of road traffic noise generated by the 500 traffic flow/distance scenarios that represent the population of acoustic conditions found near roadways. It can be seen that the different parameter sets result in a wide variation in terms of the number of noise events detected. With a few parameter value combinations, almost no events are detected, whereas in other cases, up to a mean of 77 noise events per hour are detected across the 500 scenarios. This leads to a key observation, viz that the count of noise events caused by road traffic can be very different depending on which detection algorithm is utilized-hence the importance of examining the generalized algorithm with different parameter settings.

To have face value as a potential indicator, an algorithm needs to detect road traffic noise events for many (not necessarily all) of the possible traffic flow and distance scenarios. The algorithm should also result in variation in the number of events detected as the traffic flow and/or the distance to the road changes. These conditions are met using most parameter sets, as most cells of Table 4 have non-zero mean number of noise events detected per hour, and a high correlation (Pearson $r=0.88$ ) is found between mean and standard deviation (not shown in Table 4) across the 500 scenarios examined. Some parameter value combinations result in the detection of a mean number of noise events of 10 or less across the 500 scenarios, but many combinations result in means of 20 to 60 events per hour. Low mean values tend to be associated with low standard variation in the number of noise events detected, and means greater than about 20 events with standard deviations of 20 to 80 events. Therefore, it seems reasonable to suggest that indicators should detect a mean number of noise events, across all 500 traffic and distance scenarios, of at least 20 events per hour. Note that this criterion is applied to the mean number of noise events detected across all 500 scenarios which covered the traffic and distance values as shown in Figure 2. Algorithm parameter sets excluded based on this criterion are shaded in red in Table 4 . Notably, over $60 \%$ of the closed-window algorithm parameter sets are excluded, as are all parameter combinations that employ a $30 \mathrm{~s}$ minimum gap between successive events (other than the $3 \mathrm{~dB}(\mathrm{~A})$ and $5 \mathrm{~dB}(\mathrm{~A})$ exceedances above $\left.L_{\text {Aeq }}\right)$. 


\subsection{Reliability}

The reliability of an algorithm can be assessed by examining the variation in the number of noise events detected across the 30 replications of the one-hour simulations for each scenario. The numbers in parentheses in each cell of Table 4 show the standard deviation in the number of noise events detected in the replications, pooled across the 500 scenarios. The standard deviations range from 0.3 events to 9.4 events across all algorithm parameter sets. As an initial cut, a standard deviation of less than 5.0 is suggested as being sufficiently reliable for practical measurement of the number of noise events (for a standard deviation of 5.0, some $90 \%$ of replicated event detections would be within \pm 8 events of the true value; for a standard deviation of 3.0 , some $90 \%$ of replicated event detections would be within \pm 5 events). In Table 4, those parameter sets that result in event counts regarded as unreliable are shown with blue shading. Notably, this has resulted in exclusion of near $40 \%$ of the adaptive-threshold parameter sets. Note that, as the range of scenarios considered is the same for all algorithm parameter sets, performing the analysis on the basis of the relative variability rather than the standard deviation would have resulted in the same ordering of the algorithm parameter sets in terms of their reliability.

Validity and reliability criteria filter out 107 of the potential algorithm parameter sets. Table 4 shows, in the unshaded cells, the 45 prospective algorithm parameter sets that remain for further consideration (27 open-window, 18 closed-window).

\section{Algorithm parameter set reduction}

\subsection{Clustering of algorithm parameter sets}

Given the systematic variation of parameter values in the formulation of the generalized algorithm, it can be expected that subsets of these will still exhibit considerable redundancy in their counts of noise events. To explore this, a statistical data reduction procedure may be applied to identify a smaller, more manageable, subset of the 45 valid and reliable algorithm parameter sets carried forward.

However, the variation in the number of events detected by different algorithm formulations in Table 4 is not always intuitive, as varying the parameters of the generalized algorithm can sometimes have complex effects on event detection. An overview of this complexity is illustrated graphically in Figure 4 which shows scatterplots of the number of events detected by the generalized algorithm applied with pairs of parameter sets in a random sample of ten of the 45 parameter sets. Each dot in the scatterplots represents one of the 500 scenarios modelled. Figure 4 is not intended for detailed examination of the relationship between variables, or of the number of noise events detected by them. However, the matrix does allow the observation that the relationships between the number of events detected by the different algorithm formulations across the 500 scenarios are, for the most part, elaborate and non-linear. This is an important observation, as the absence of linearity influences the procedures appropriate to reduce redundancy.

As many of the algorithm parameter sets result in non-linear inter-relationships between the number of noise events detected, examining redundancy using bivariate correlations was not possible. Principal component analysis also requires linearity between variables. Instead, the Categorical Principal Component Analysis (CATPCA) procedure implemented in SPSS 25 was utilized, as it allows data reduction between variables with non-linear relationships. The data set analyzed consisted of the number of noise events returned from the generalized algorithm applied, with all 45 selected parameter sets, to each of the 500 traffic/distance scenarios. A two-dimension solution of the CATPCA analysis explained $81.7 \%$ of the total variance in the variables, and the component loadings of the 45 parameter sets (Varimax rotated) on these dimensions are shown in Figure 5(a). Seven clusters of parameter sets that have similar loadings on both dimensions are identified by broken circles in Figure 5(a). The parameter sets that constitute the seven clusters are listed in Table 5.

\subsection{Relationships between parameter sets within clusters}

The nature of the CATPCA analysis is that each cluster will encompass much of the redundancy between parameter sets. Bivariate scatterplots can then be used to examine selected relationships between parameter sets within clusters, particularly between similar parameter sets differentiated by just one dimension in their formulation, e.g. open vs closed windows, different minimum gap between successive events, and different exceedance thresholds. Several of these relationships are discussed below, and provide useful insight into the effects of varying parameters in the generalized noise event detection algorithm. 


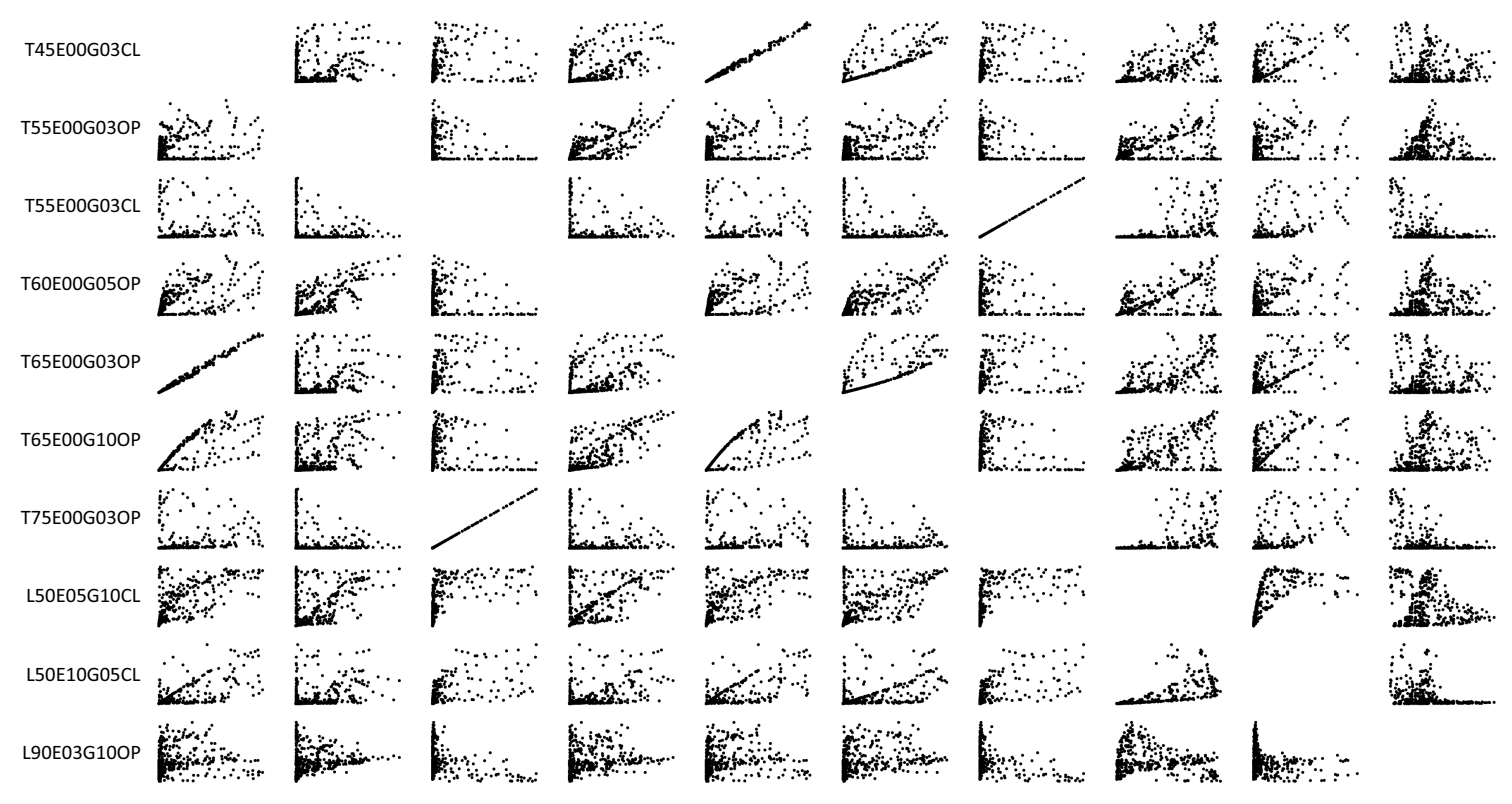

Figure 4: Scatterplot matrix of the number of noise events detected by the generalized noise event detection algorithm, for 10 parameter sets, selected randomly out of the 45 parameter sets shown unshaded in Table 4. Each of the 500 dots in each panel represents a single scenario. Both $x$ and $y$ axes in each plot denote the number of noise events detected by the generalized algorithm with the respective parameter set, but the scale varies for each (from 0-80 to 0-350 noise events).
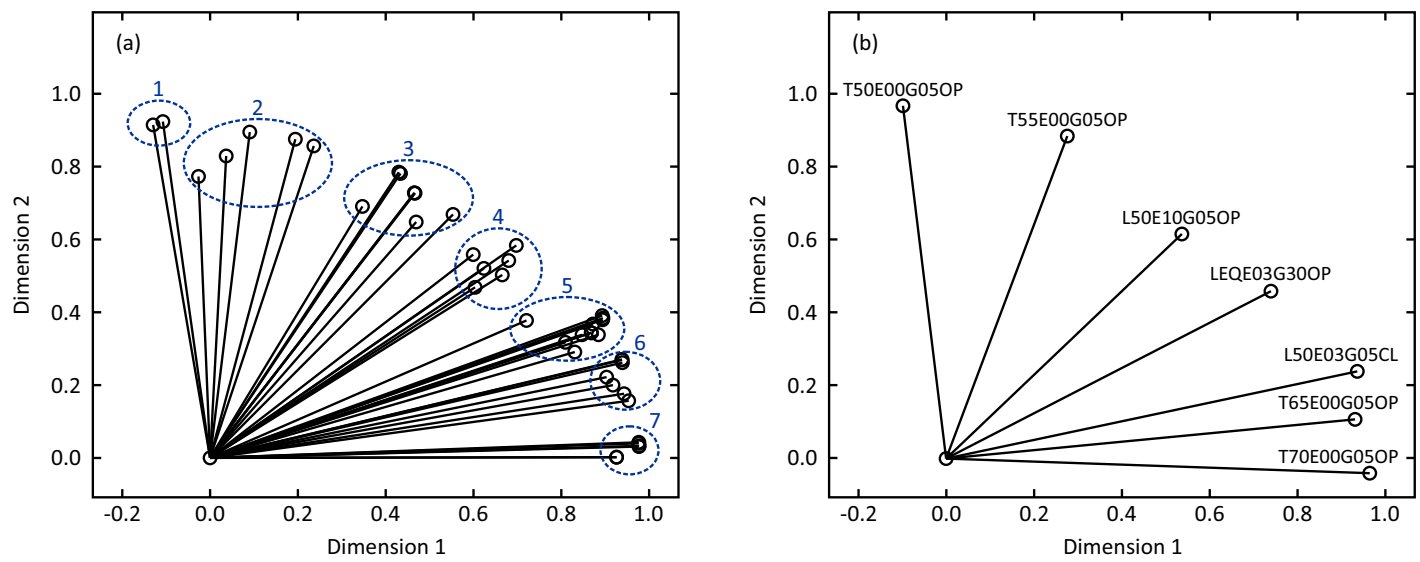

Figure 5: Varimax rotated component loadings on two dimensions from the CATPCA analysis, on the basis of (a) the 45 selected parameter sets as presented in Table 4 (non-shaded), and (b) the seven parameter sets chosen as representative of those in the clusters, as show in panel (a) with a blue circle. 
Table 5: The parameter sets that constitute the seven clusters as shown in Figure 5. The prototype parameter sets selected to represent the others within a cluster are shown in bold.

\begin{tabular}{ll}
\hline Cluster & Parameter sets \\
\hline 1 & T50E00G05OP, T50E00G03OP \\
2 & T55E00G05OP, L90E05G10OP, L90E03G10OP, T55E00G10OP, T55E00G03OP \\
3 & L50E10G05OP, L50E15G03OP, L90E15G10OP, L90E15G05OP, L50E10G10OP, \\
& L50E10G03OP, T60E00G10OP, L90E10G10OP \\
4 & LEQE03G30OP, T60E00G05OP, L90E03G10CL, T60E00G03OP, L50E05G10OP, \\
& LEQE05G30OP \\
5 & L50E03G05CL, L50E05G05CL, L50E05G10CL, LEQE05G10CL, L50E03G10OP, \\
& L50E03G10CL, L50E10G03CL, L50E10G05CL, L90E05G10CL, T45E00G10CL, \\
& T65E00G10OP \\
& T65E00G05OP, L90E10G03CL, L90E10G05CL, L90E10G10CL, T45E00G05CL, \\
& T45E00G03CL, T65E00G03OP \\
& T70E00G05OP, T50E00G05CL, T50E00G03CL, T70E00G03OP, T55E00G03CL, \\
& T75E00G03OP \\
&
\end{tabular}

\subsubsection{Open-window vs closed-window}

Clusters 5 to 7 in Table 5 include parameter sets that employ a fixed threshold, for both open and closed window conditions. Given the $20 \mathrm{~dB}(\mathrm{~A})$ difference between open and closed windows used in the modelling, it is unsurprising that the 45,50 and $55 \mathrm{~dB}(\mathrm{~A})$ closed-window fixed-threshold cases are found to have strong linear relationships with the 65,70 and $75 \mathrm{~dB}(\mathrm{~A})$ open-window fixed-threshold cases respectively, and thus do not provide information dissociated from that generated by open-window cases. Given that none of the other (60 to $75 \mathrm{~dB}$ (A) fixed-threshold) closedwindow parameter sets result in an algorithm that produces sufficient counts to be used as noise event indicator (Table 4), the consequence is that fixed-threshold parameter sets under closed-window conditions do not need to be considered further for potential road traffic noise event indicators. However, adaptive-threshold parameter sets, based on $L_{\mathrm{A} 50}$ and $L_{\mathrm{A} 90}$ under closed window conditions, remain as candidates.

\subsubsection{Effect of time gaps between successive events}

Each cluster in Table 5 includes parameter sets that differ in formulation only in terms of the minimum time gap between successive events. Figure 6, panels (a) and (b), shows scatterplots of the number of events detected using the open-window $55 \mathrm{~dB}(\mathrm{~A})$ fixed-threshold parameter sets with three different minimum time gaps. As could be expected, increasing the minimum gap between successive events results in fewer events being detected, with T55E00G03OP detecting more events than T55E00G05OP, and both in turn detecting more than T55E00G10OP. Figure 6 shows that, while pairs of these parameter sets are strongly related, the longer gap is also associated with some drop-out in event detection for some traffic and distance scenarios, resulting in a smaller number of noise events. Fixed-threshold parameter sets at other levels produced similar results, as did adaptive-threshold parameter sets based on $L_{\mathrm{A} 50}$ and $L_{\mathrm{A} 90}$, though with the drop-out occurring at high numbers of events, as shown in Figure 6, panels (c) and (d). This means that, for otherwise similarly formulated parameter sets, different time gaps will tend to produce different, but correlated, numbers of noise events. Redundancy in the parameter sets considered can thus be reduced, where there are similar parameter sets differentiated by time gap, by utilizing just one of the time gaps.

\subsubsection{Effect of emergence requirements in adaptive threshold parameter sets}

An example of the effect of increasing the emergence required above an adaptive threshold is illustrated in Figure 7 , which shows the number of noise events detected by the generalized algorithm using three different closed-window parameter sets, differentiated only by different emergences of 3,5 or $10 \mathrm{~dB}(\mathrm{~A})$ above $L_{\mathrm{A} 50}$. As could be anticipated, the greater the required emergence, the fewer noise events are detected-but, again, the counts of events using the different parameter sets are related. The spread of the number of noise events detected also increases as the emergence increases, becoming quite large for higher numbers of events. Redundancy in the parameter sets considered can thus 

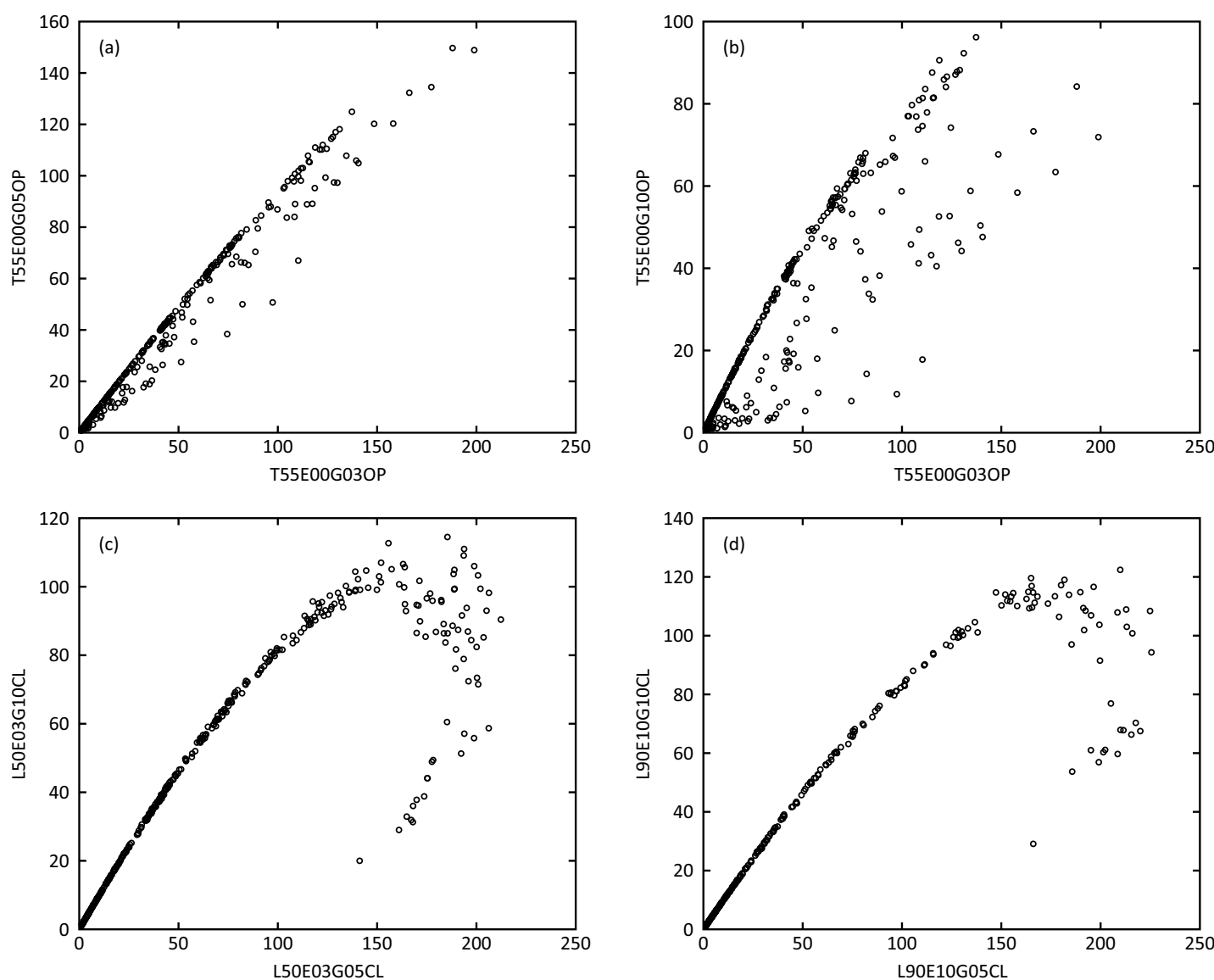

Figure 6: Scatterplots illustrating the largely linear relationships between the number of noise events detected using the generalized algorithm with (a) the open-window $55 \mathrm{~dB}$ (A) fixed-threshold parameter sets with minimum time gaps of 3 and $5 \mathrm{~s}$ between events, (b) the open-window $55 \mathrm{~dB}$ (A) fixed-threshold parameter sets with minimum time gaps of 3 and $10 \mathrm{~s}$ between events, (c) the closed-window $3 \mathrm{~dB}(\mathrm{~A})$ emergence above $L_{\mathrm{A} 50}$ parameter sets with minimum time gaps of 5 and $10 \mathrm{~s}$ between events, and (d) the closed-window $10 \mathrm{~dB}(\mathrm{~A})$ emergence above $L_{\mathrm{A} 90}$ parameter sets with minimum time gaps of 5 and 10 s between events. 

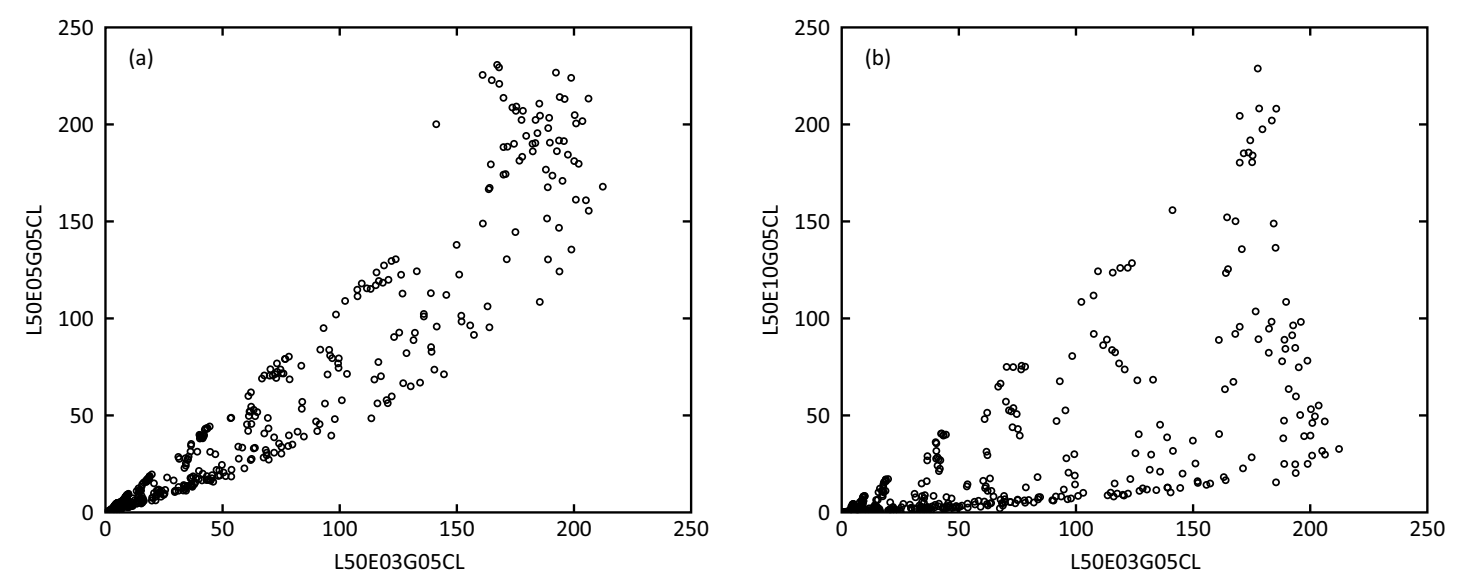

Figure 7: Scatterplots illustrating the relationships between the number of noise events detected using the generalized algorithm with the closedwindow adaptive-threshold parameter sets with minimum time gap of $5 \mathrm{~s}$ between events, and with (a) exceedance of 3 and $5 \mathrm{~dB}(\mathrm{~A})$ above $L_{\mathrm{A}} 50$, and (b) exceedance of 3 and $10 \mathrm{~dB}(\mathrm{~A})$ above $L_{\mathrm{A} 50}$.

be reduced, where there are similar parameter sets differentiated by emergence, by utilizing just one of the emergence levels.

\subsubsection{Relationships between other parameter sets within clusters}

Figure 8 shows the relationships between the number of noise events detected by the generalized algorithm using various other pairs of parameter sets from within the same clusters. This includes between fixed-threshold and adaptive-threshold parameter sets, and between adaptive-threshold parameter sets that use different noise indicators as thresholds. From the 500 data points on each of the plots in Figure 8 (each representing one traffic/distance scenario) it can be seen that the number of noise events detected can be very different for different parameter sets, and that the patterns tend to be different with each pair-some with considerable scatter and with many outliers. The patterns in each of the six plots in Figure 8 confirm that there is a discernible relationship between most of the pairs of parameter sets shown, and of sufficient strength of association to explain why the CATPCA analysis allocated each of the pairs to the same cluster of variables.

\subsection{Selection of representative parameter sets}

Based on the examination in Section 5.2 of the relationships between parameter sets within the seven clusters, a single parameter set has been selected as representative of those in each cluster. These parameter sets are shown in bold type in Table 5. The choice of representative parameter sets was based on the following: open-window fixedthreshold parameter sets were chosen over closed-window fixed-threshold parameter sets; parameter sets using a $5 \mathrm{~s}$ minimum time gap between successive events were retained in favor of those using longer or shorter time gaps within the same cluster; adaptive-threshold parameter sets with the smallest emergence were chosen above those with larger emergence threshold; and finally, where choice still remained, the type of parameter set most commonly represented within the cluster was selected-or in the case of Cluster 4, the type of parameter set (emergence above an adaptive threshold based on $L_{\mathrm{Aeq}}$ ) not included in any other cluster. We emphasize that any of the other parameter sets within each cluster in Figure 5 and Table 5 (some of which have been used by others in previous studies) could, equally, have been nominated as the cluster representative.

This data reduction to seven prototype parameter sets does not imply that the other parameter sets in Table 5 are not appropriate to use in future practice: they still meet the requirements of valid and reliable measures of noise events. Note again that determination of the best algorithm to utilize for event counting will eventually have to be based on studies on human perception of events. For example, while the choice for a $5 \mathrm{~s}$ minimum time gap may seem arbitrary, the number of noise events, as detected with an algorithm employing a particular minimum time gap, could subsequently be increased/decreased if this is suggested from human-response studies, by the use of a detection 

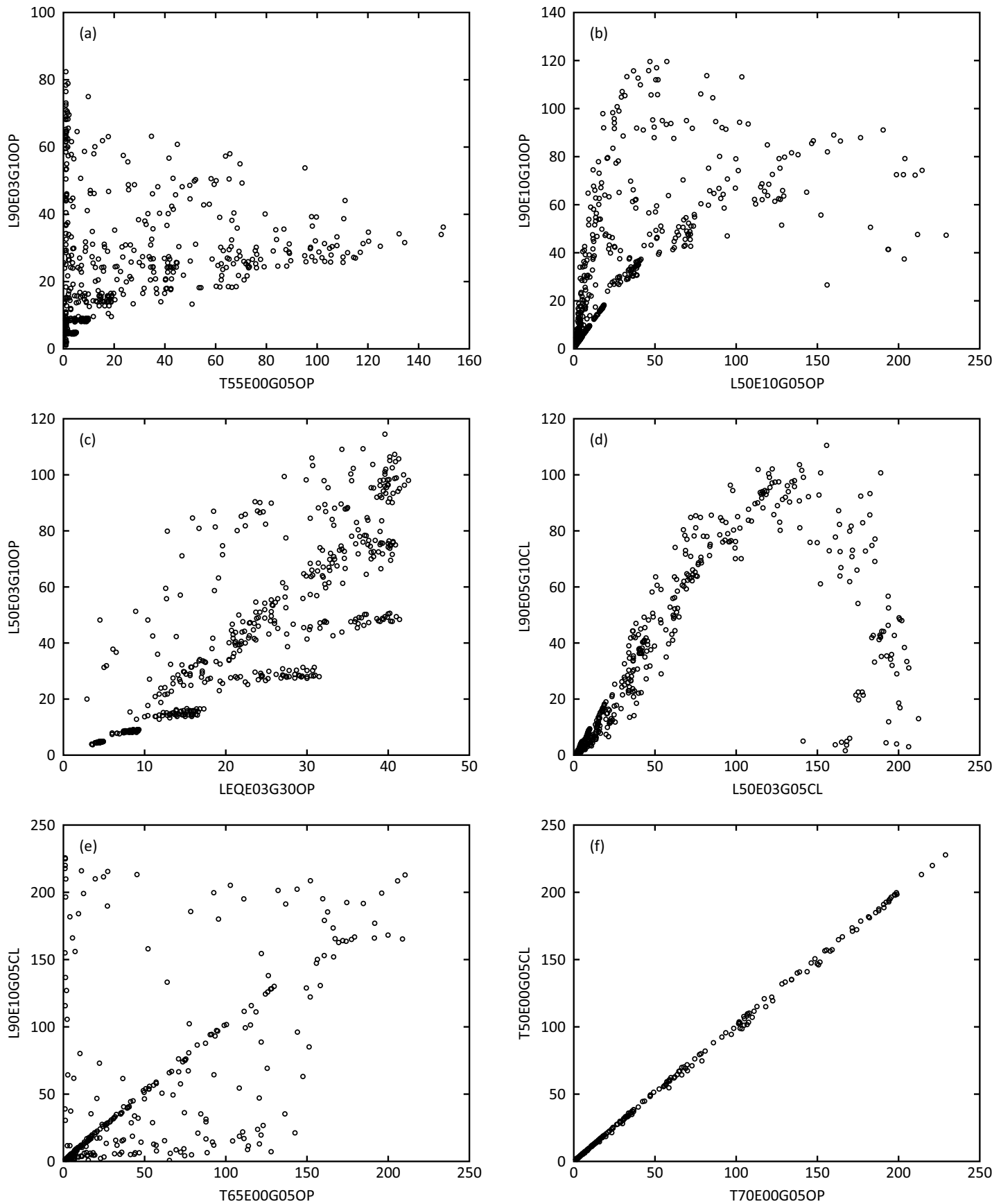

Figure 8: Scatterplots illustrating the relationships between the number of noise events detected by the generalized algorithm using pairs of parameter sets within the clusters in Table 5: (a) Cluster 2, (b) Cluster 3, (c) Cluster 4, (d) Cluster 5, (e) Cluster 6, (f) Cluster 7. 


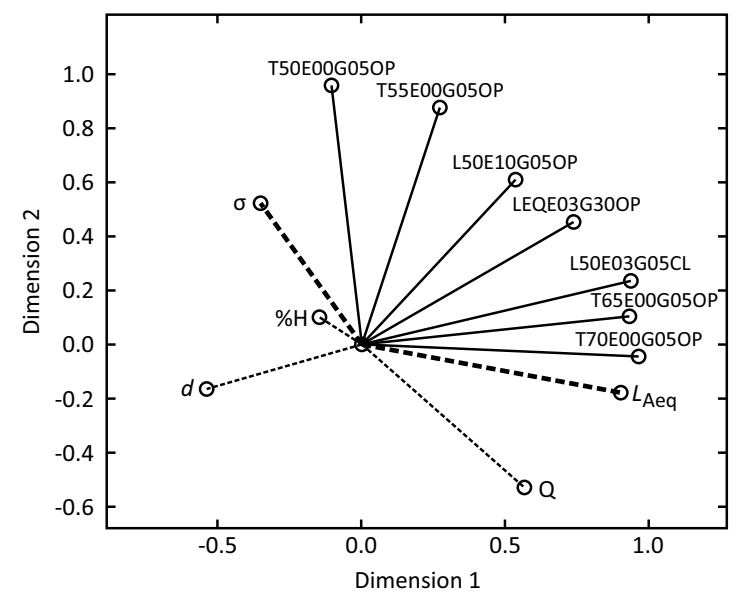

Figure 9: Varimax rotated component loadings on two dimensions from the CATPCA analysis for the seven representative parameter sets, the same as in Figure 5, but with three supplementary scenario variables (total traffic flow $Q$, percentage of heavy vehicles $\% \mathrm{H}$, and distance $d$ to the road) and two supplementary acoustic variables (energy-equivalent level $L_{\mathrm{Aeq}}$ and standard deviation $\sigma$ of the sound level outside the dwelling), also loaded on the two-dimension solution.

algorithm with longer/shorter time gap. This data reduction to a smaller set has only been done to generate a more manageable number of parameter sets for the generalized noise event detection algorithm, which could be used for later analysis of acoustic conditions under which noise event measures may be useful.

It is possible to examine this set of representative parameter sets further, by running the CATPCA procedure again, using only the seven representative parameter sets. The outcome is the two dimension solution, Varimax rotated, shown in Figure 5(b). The two dimensions account for $84.9 \%$ of the total variance in the seven variables, which are widely separated on the two dimensions of Figure 5. By including supplementary variables, some interpretation of the two dimensions of the CATPCA solution can be provided. Figure 9 shows the CATPCA solution in which three traffic and sound propagation variables (total traffic flow $Q$, percentage of heavy vehicles $\% \mathrm{H}$, and distance $d$ to the road) and two acoustic variables (energy-equivalent level $L_{\text {Aeq }}$ and standard deviation $\sigma$ of the sound level outside the dwelling) were included as supplementary variables (these do not enter the analysis itself but only facilitate interpretation). From Figure 9, Dimension 1 can be interpreted as the overall level of the road traffic noise, because $L_{\text {Aeq }}$ (the outside energy equivalent sound level) loads highly on that dimension. Dimension 2 is orthogonal to Dimension 1 and thus largely unrelated to the overall sound level. However, the standard deviation $\sigma$ of the sound level outside the dwelling, loads reasonably high on it. This second dimension can therefore be partially interpreted as the variability of the sound level outside the dwelling caused by traffic. Interpretation of the two dimensions in terms of road traffic parameters and distance conditions is not so straightforward, with each of the variables $Q, \% \mathrm{H}$ and $d$ loading on both dimensions, though $d$ does load predominantly, and as would be expected, negatively, on Dimension 1-the overall level of road traffic noise.

The interpretation of the two dimensions in Figure 9 facilitates interpretation of the various clusters of eventdetection parameter sets. Clusters 1 and 2 (represented by the 50 and $55 \mathrm{~dB}(\mathrm{~A})$ fixed-threshold parameter sets, open window) are associated with higher variability of the road traffic noise signal than they are with the overall level of traffic noise. Clusters 5, 6 and 7 (represented by the $L_{\mathrm{A} 50}+3 \mathrm{~dB}(\mathrm{~A})$ adaptive-threshold, closed window, and the 65 and $70 \mathrm{~dB}$ (A) fixed-threshold parameter sets, open windows) are associated with high overall levels rather than the variability of the signal. Clusters 3 and 4 (both represented by adaptive-threshold parameter sets) are interim between these, being associated with both level and variability of the road traffic noise signal. Broadly, this suggests that the number of noise events detected by using different parameter sets is differentially associated with overall level of the traffic noise and with the variability of the road traffic signal. Figure 10, which shows the number of noise events per hour detected using each of the seven representative parameter sets, as a function of traffic demand and distance to the roadway, reflects this finding: the traffic demand range in which the detected number of noise events varies more or less linearly with the traffic demand gradually shifts to higher values, from cluster 1 to cluster 7 . At any 
particular distance from a roadway, the level of the road traffic noise will increase monotonically as the traffic flow increases. However, the variability of a traffic noise signal will initially increase as traffic flow increases from zero, but then decrease at the higher flow rates-the troughs between successive peaks in the traffic noise signal fill in, hence resulting in lower variability. By contrast, both level and variability of a traffic noise signal decrease with increasing distance from the roadway. These complex interactions between level and variability of the road traffic noise signal suggest that a detailed analysis of the behaviour of different noise event detection models across the population of acoustic conditions near roadways will be necessary.

\section{Conclusions}

This paper has explored a generalized algorithm to detect noise events in the time history of the sound level caused by road traffic streams. This generalized algorithm was rigorously developed from the literature, and is based on fixed thresholds or on emergence above adaptive thresholds of $L_{\mathrm{Aeq}}, L_{\mathrm{A} 50}$ and $L_{\mathrm{A} 90}$. Threshold level, emergence, and minimum time gap between successive events were varied. This resulted in 76 different noise event detection protocols, applicable to both window-open and window-closed conditions. These parameter value combinations were systematically applied to detect events in simulated time histories of road traffic noise across a population of acoustic conditions found near roadways; 500 different scenarios were considered with varying traffic flow, vehicle mix, speed limit and distance to the roadway.

An appropriate parameter set for the noise event detection algorithm will eventually have to be selected through human effects research by examining if, and which, noise event measures of a road traffic noise signal correlate best with human response to that signal. However, any method used to detect and count the events in road traffic noise must first produce stable estimates of the number of events, and should vary sensibly as the traffic and distance conditions change. It was shown that the number of noise events detected in the time history of the sound level caused by road traffic is highly dependent on the parameters utilized-justifying a study of this nature into alternative formulations of detection algorithms as a step towards identification of appropriate algorithm(s) which use could eventually be standardized.

About $20 \%$ of the detection parameter sets resulted in an unreliable algorithm, resulting in different numbers of events counted for traffic noise signals that are generated by identical traffic and propagation conditions. Such unstable measures would not be suitable for use as limit criteria. It was also found that approximately $50 \%$ of the parameter sets did not give rise to an algorithm that results in a reasonable range of numbers of noise events when applied across all acoustic conditions. Excluding these, 45 reliable and functional variations on the generalized exceedance algorithm remained. Subsequent application of dimension-reduction procedures to reduce redundancy between these identified seven that were representative of the original set. The numbers of noise events detected with the generalized algorithm through the application of several of these parameter sets were found to be associated with the overall road traffic noise level, whereas other parameter sets were associated more with the variability of the road traffic noise. This small number of noise event detection parameter sets parsimoniously represents the large number of possible exceedance indicators developed from those described in literature, but has been compiled without the loss of information that could occur through arbitrary discard of any particular detection formulation. The identification of this small subset of parameter value combinations of a generalized noise event detection algorithm is an important step towards further work to evaluate the acoustic conditions under which event counting in road traffic noise signals may be appropriate.

\section{Acknowledgments}

The authors gratefully acknowledge the assistance provided through the inaugural grant from the Australian Acoustical Society Research Grants Program for the conduct of this work.

\section{References}

[1] Fidell S, Pearsons K, Tabachnick BG, Howe R. Effects on sleep disturbance of changes in aircraft noise near three airports. J Acoust Soc Am 2000;107(5):2535-47. 

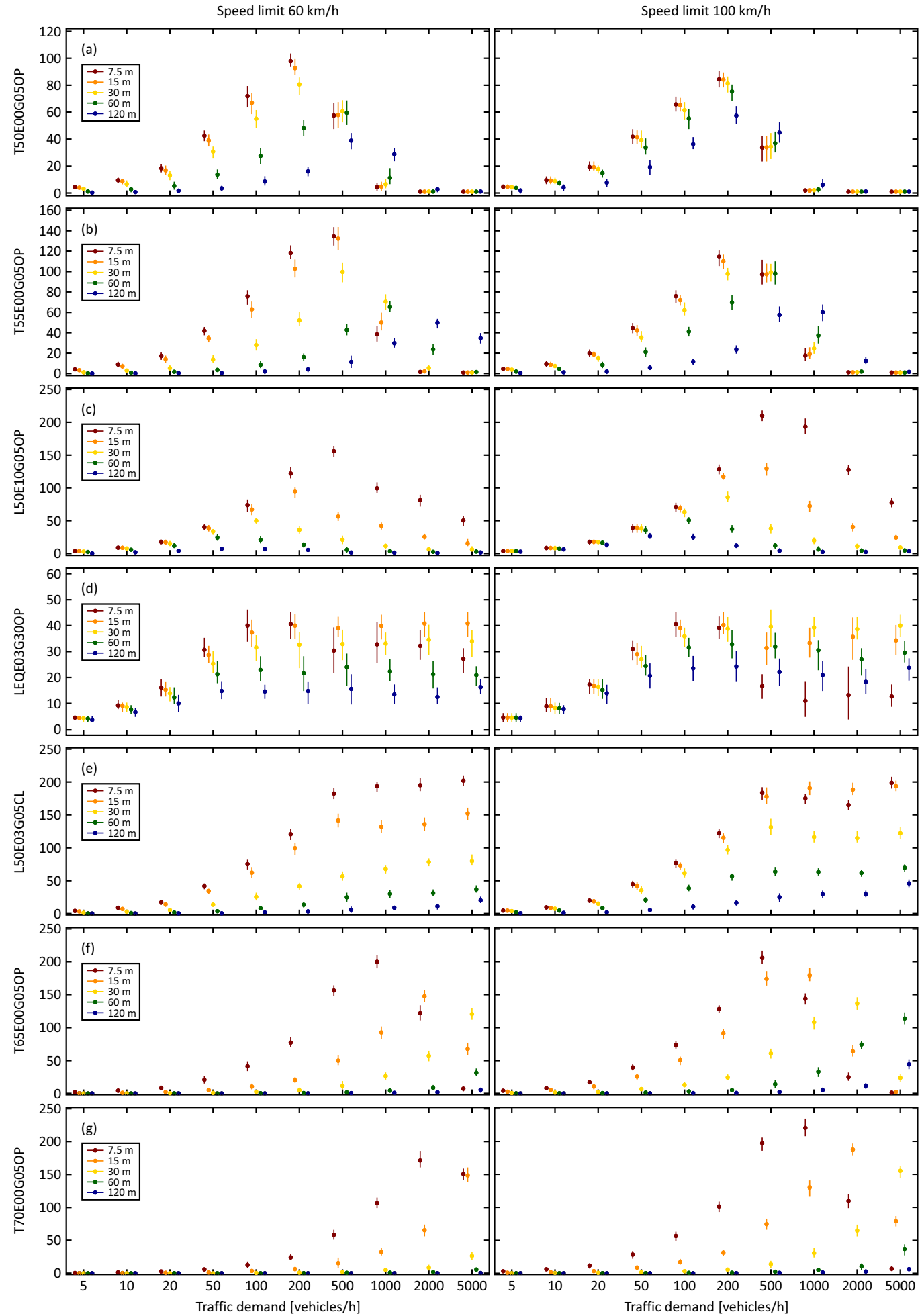

Figure 10: Number of noise events per hour detected by the generalized algorithm using each of the seven representative parameter sets (panels ordered according to cluster number), as a function of the traffic demand and the distance to the roadway, for speed limits of $60 \mathrm{~km} / \mathrm{h}$ (left panels) and $100 \mathrm{~km} / \mathrm{h}$ (right panels), and for $10 \%$ heavy vehicles. The dots represent the mean value over the 30 replications for each scenario; the vertical lines represent the range that contains $90 \%$ of the outcomes of the 30 replications for each scenario (i.e. from the 5-percentile to the 95 -percentile of the number of events). 
[2] Passchier-Vermeer W, Vos H, Steenbekkers JHM, van der Ploeg FD, Groothuis-Oudshoorn K. Sleep disturbance and aircraft noise exposureexposure-effect relationships. TNO Report 2002.027; TNO Prevention and Health; Leiden, The Netherlands; 2002.

[3] Couvreur L, Laniray M. Automatic noise recognition in urban environments based on artificial neural networks and hidden Markov models. In: Proc. Inter-noise. Prague, Czech Republic; 2004, p. 555-62.

[4] Griefahn B, Bröde P, Marks A, Basner M. Autonomic arousals related to traffic noise during sleep. Sleep 2008;31(4):569-77.

[5] Basner M, Müller U, Griefahn B. Practical guidance for risk assessment of traffic noise effects on sleep. Appl Acoust 2010;71(6):518-22.

[6] Treagus R, Beazley CJ. Noise monitoring program for assessing the impact of maximum traffic noise levels. In: Proc. Acoustics'05. Busselton, Australia; 2005, p. 271-6.

[7] Beaumont J, Semidor C. Interacting quantities of the soundscape due to transport modes. In: Proc. Inter-noise. Rio de Janeiro, Brazil; 2005, p. 3408-14

[8] Can A, Leclercq L, Lelong J, Defrance J. Capturing urban traffic noise dynamics through relevant descriptors. Appl Acoust 2008;69(12):1270-80.

[9] Öhrström E, Björkman M, Rylander R. Laboratory annoyance and different traffic noise sources. J Sound Vib 1980;70(3):333-41.

[10] Fields JM. The effect of numbers of noise events on people's reactions to noise: an analysis of existing survey data. J Acoust Soc Am 1984;75(2):447-67.

[11] Öhrström E, Rylander R, Björkman M. Effects of night time road traffic noise — an overview of laboratory and field studies on noise dose and subjective noise sensitivity. J Sound Vib 1988;127(3):441-8.

[12] Suzuki S, Kawada T, Kiryu Y, Sasazawa Y, Tamura Y. Transient effect of the noise of passing trucks on sleep EEG. J Sound Vib 1997;205(4):411-5.

[13] Samuels SE, Parnell J. Peak noise events occurring in road traffic noise. In: Proc. Acoustics'04. Gold Coast, Australia; 2004, p. 307-12.

[14] Heimann D, Schady A. Derivation of sound-level characteristics to assess traffic development scenarios. Appl Acoust 2016;103:1-9.

[15] Campbell JA, Parnell J. A vehicle maximum noise level study. In: Proc. Acoustics'04. Gold Coast, Australia; 2004, p. 501-6.

[16] Mitchell P. Speed and road traffic noise-The role that lower speeds could play in cutting noise from traffic. Technical report; UK Noise Association; Kent, UK; 2009.

[17] De Muer T, Botteldooren D, De Coensel B, Berglund B, Nilsson ME, Lercher P. A model for noise annoyance based on notice-events. In: Proc. Inter-noise. Rio de Janeiro, Brazil; 2005, p. 3323-32.

[18] De Coensel B, Botteldooren D, De Muer T, Berglund B, Nilsson ME, Lercher P. A model for the perception of environmental sound based on notice-events. J Acoust Soc Am 2009;126(2):656-65.

[19] Roberts MJ, Western AW, Webber MJ. A theory of patterns of passby noise. J Sound Vib 2003;262(5):1047-56.

[20] Brink M, Basner M, Schierz C, Spreng M, Scheuch K, Bauer G, et al. Determining physiological reaction probabilities to noise events during sleep. Somnologie 2009;13:236-43.

[21] Brown AL. An overview of concepts and past findings on noise events and human response to surface transport noise. In: Proc. Inter-noise. Melbourne, Australia; 2014, p. 1925-32.

[22] European Commission. Directive 2002/49/EC of the European Parliament and of the Council relating to the assessment and management of environmental noise. 2002.

[23] Night noise guidelines for Europe. Tech. Rep.; World Health Organization Regional Office for Europe; Copenhagen, Denmark; 2009.

[24] Jones K, Cadoux R. Metrics for aircraft noise. ERCD Report 0904; Environmental Research and Consultancy Department, Civil Aviation Authority; London, UK; 2009.

[25] Aasvang GM, Øverland B, Ursin R, Moum T. A field study of effects of road traffic and railway noise on polysomnographic sleep parameters. J Acoust Soc Am 2011;129(6):3716-26.

[26] Griefahn B, Marks A, Robens S. Experimental shift work and noise exposure during sleep. In: Proc. ICBEN. London, UK; 2011 , p. 595-602.

[27] Brown AL. Issues in the measurement of sleep-related noise events in road traffic streams. In: Proc. Acoustics'13. Victor Harbor, Australia; 2013, p. 1-6.

[28] Makarewicz R, Gołębiewski R. The uncertainty of noise composed of separate sound events. Arch Acoust 2016;41(1):133-8.

[29] Murray BJ. Assessment of road traffic maximum noise events at night time. In: Proc. Acoustics'01. Canberra, Australia; 2001 , p. 1-5.

[30] Taylor SM, Hall FL, Birnie SE. Transportation noise annoyance: testing of a probabilistic model. J Sound Vib 1987;117(1):95-113.

[31] Hall FL, Taylor SM, Birnie SE. Activity interference and noise annoyance. J Sound Vib 1985;103(2):237-52.

[32] Lambert J, Champelovier P, Vernet I. Annoyance from high speed train noise: a social survey. J Sound Vib 1996;193(1):21-8.

[33] Fidell S, Pearsons K, Tabachnick B, Howe R, Silvati L, Barber DS. Field study of noise-induced sleep disturbance. J Acoust Soc Am 1995;98(2):1025-33.

[34] Müller U, Basner M, Plath G, Samel A. Aircraft noise effects on sleep: acoustical setup and results of DLR laboratory and field studies. In: Proc. Inter-noise. Prague, Czech Republic; 2004, p. 2670-7.

[35] Mietlicki F, Ribeiro C, Sineau M. Noise generated by the Paris ring-road: state of knowledge and issues. In: Proc. Inter-noise. Innsbruck, Austria; 2013, p. 2815-24.

[36] Wunderli JM, Pieren R, Habermacher M, Vienneau D, Cajochen C, Probst-Hensch N, et al. Intermittency ratio: a metric reflecting short-term temporal variations of transportation noise exposure. J Expo Sci Environ Epidemiol 2015;:1-11.

[37] Campbell JA, Isles S. RTA Environmental Noise Management Manual. Haymarket, NSW, Australia: Roads and Traffic Authority of New South Wales; 2001.

[38] De Coensel B, Botteldooren D. The quiet rural soundscape and how to characterize it. Acta Acust Acust 2006;92(6):887-97.

[39] Tulen JHM, Kumar A, Jurriëns AA. Psychophysiological acoustics of indoor sound due to traffic noise during sleep. J Sound Vib 1986;110(1):129-41

[40] Liguori C, Paolillo A, Ruggiero A, Russo D. Outlier detection for the evaluation of the measurement uncertainty of environmental acoustic noise. IEEE Trans Instrum Meas 2016;65(2):234-42.

[41] Ribeiro C, Anselme C, Mietlicki F, Vincent B, Da Silva R. At the heart of Harmonica project: the Common Noise Index (CNI). In: Proc. Inter-noise. Innsbruck, Austria; 2013, p. 4088-97. 
[42] Sato T, Yano T, Björkman M, Rylander R. Road traffic noise annoyance in relation to average noise level, number of events and maximum noise level. J Sound Vib 1999;223(5):775-84.

[43] Marks A, Griefahn B, Basner M. Event-related awakenings caused by nocturnal transportation noise. Noise Control Eng J 2008;56(1):52-62.

[44] Bite P, Augusztinovicz F, Flindell IH. Unexpectancy in environmental noise assessment. In: Proc. Forum Acusticum. Budapest, Hungary; 2005, p. 1831-5.

[45] Gjestland T, Oftedal G. Assessment of noise annoyance: The introduction of a threshold level in $L_{\mathrm{eq}}$ calculations. J Sound Vib 1980;69(4):603-10

[46] De Coensel B, Brown AL, Tomerini D. A road traffic noise pattern simulation model that includes distributions of vehicle sound power levels. Appl Acoust 2016;111:170-8.

[47] Peeters B, van Blokland G. The noise emission model for European road traffic. Technical Report — Deliverable 11 of the Imagine project IMA55TR-060821-MP10; M+P Consulting Engineers; Vught, The Netherlands; 2007.

[48] Brown AL, Tomerini D. Distribution of the noise level maxima from the pass-by of vehicles in urban road traffic streams. Road Transp Res 2011;20(3):50-63.

[49] ISO 9613-Part 2. Acoustics - Attenuation of sound during propagation outdoors - Part 2: General method of calculation. International Standard ISO 9613-2; International Organization for Standardization; Geneva, Switzerland; 1996. 